\title{
A nutriepigenetic pathway links nutrient information to sensory plasticity
}

\author{
Anoumid Vaziri ${ }^{1,2}$, Daniel Wilinski ${ }^{1}$, Peter L. Freddolino ${ }^{3,4}$, Carrie R. Ferrario ${ }^{5}$, Monica Dus ${ }^{1,2, *}$
}

${ }^{1}$ The Molecular, Cellular and Developmental Biology Graduate Program, The University of Michigan, Ann Arbor, MI 49109

${ }^{2}$ Department of Molecular, Cellular and Developmental Biology, College of Literature, Science, and the Arts, The University of Michigan, Ann Arbor, MI 49109

${ }^{3}$ Department of Biological Chemistry, The University of Michigan Medical School, Ann Arbor, MI 48109, USA

${ }^{4}$ Department of Computational Medicine and Bioinformatics, The University of Michigan Medical School, Ann Arbor, MI 48109, USA

${ }^{5}$ Department of Pharmacology, and Psychology Department (Biopsychology) The University of Michigan, Ann Arbor, MI 48109, USA

*Correspondence: mdus@umich.edu

\section{Keywords}

Nutriepigenetics, metabolism, sensory adaptations, sugar, obesity, 


\section{Abstract}

Diet composition has a profound influence on brain physiology and behavior, but the mechanisms through which nutrient information is transmuted into neural changes remain elusive. Here we uncover how the metabolic enzyme O-GIcNAc Transferase (OGT) transforms information about the dietary environment into taste adaptations. We show that in the fly $D$. melanogaster, OGT decorates the chromatin of the sweet taste neurons and provides the nutrient context to drive changes in chromatin accessibility in response to high dietary sugar. Specifically, we found that OGT cooperates with the epigenetic silencer Polycomb Repressive Complex 2.1 (PRC2.1) to promote nutrient-sensitive variations in chromatin openness; these chromatin dynamics result in changes in gene expression and taste plasticity that are dependent on the catalytic activity of OGT. Parallel nutrigenomic signatures were also observed in the lingual epithelium of rats exposed to high dietary sugar, suggesting that this conserved metabolic-epigenetic pathway may also underlie diet-dependent taste changes in mammals. Together our findings reveal a novel role for nutriepigenetic signaling in the brain: amplifying nutrient perturbations into robust changes in chromatin accessibility and transcriptional output that shape neural and behavioral plasticity. 


\section{Introduction}

Food provides the energy, biomass, and essential micronutrients to sustain life. In the absence of this fuel, the chaotic mechanics of life stop working, and all growth, division, and endurance halt. It is thus not surprising that much attention has been paid to the energetic function of food, especially as it relates to human health and disease. However, food does more than passively supply nourishment for biological processes: it also actively guides them. Indeed, research in the field of nutriepigenetics shows that components of food can, directly or indirectly, shape gene expression in metazoa ${ }^{1,2}$. As such, food can be thought of as a type of "information" that cells use to calibrate their responses to what is perhaps the most critical of environmental stressors: nutrient availability.

These nutrient information highways arise from the intersection of metabolic, signaling, and gene regulatory pathways, and function to amplify often feeble and transient nutrient inputs into forceful and coordinated responses across the cell ${ }^{3}$. For example, nutrient availability affects cell fate decisions, such as the balance between maintaining stemness and differentiation ${ }^{4-7}$, as well as the activation of immune cells ${ }^{8-10}$, cancer progression ${ }^{11-14}$ and aging ${ }^{15-17}$. These studies have identified key informational pathways and characterized their "rules" of communication by showing that nutrients can affect gene expression by providing substrates for enzymatic- and non-enzymatic modifications to DNA, RNA, and proteins, directly catalyzing them, serving as cofactors, or changing the localization, stability, levels, and activity of biological effectors such as mRNA and proteins ${ }^{2}$. As such, we have learnt much about how nutrients and metabolites instruct cell physiology. In contrast, very little is understood about nutriepigenetic signaling in the brain ${ }^{18}$, despite the fact that diet composition affects brain function and behavior profoundly, reviewed in ${ }^{19}$. Thus, we do not know much about which nutrient informational pathways are engaged in neurons under different dietary conditions, how they pass on specific messages, and how these, in turn, enable physiological responses, such as changes in synaptic plasticity or activity ${ }^{18,20}$. To answer some of these questions, we studied the role of nutriepigenetic mechanisms in diet-induced sweet taste plasticity, a form of sensory adaptation that develops with exposure to calorie-dense diets ${ }^{21,22}$.

In mammals and insects, different levels of sugar and fat in the diet affect sweet taste sensation by changing the responses of the taste-receptor cells, sensory nerves, and sensory regions to sweet stimuli, as reviewed in ${ }^{21-24}$. In particular, recent studies have shown that higher dietary levels of fat and sugar depress sweet taste sensation ${ }^{25-32}$. Although the molecular mechanisms are still not understood in mammals ${ }^{21,33}$, our previous work in D. melanogaster flies implicated the conserved chromatin repressor and Histone 3 Lysine 27 Methyltransferase complex Polycomb Repressive Complex 2.1 (PRC2.1) as a key regulator of diet-induced sweet taste plasticity ${ }^{30}$. Specifically, we found that the high sugar environment promoted a redistribution of PRC2.1 occupancy on the chromatin of the taste cells, which resulted in the repression of a transcriptional hub predicted to modulate the synaptic, signaling, and metabolic properties of these neurons. However, the signals 
that guided nutrient-dependent changes in PRC2.1 repression, remained unknown. Here we report that the metabolic enzyme O-GIcNAc Transferase (OGT) instructs the nutrient-dependent binding and repressive activity of PRC2.1 in the sweet taste neurons. In the absence of OGT catalytic activity, PRC2.1 occupancy loses its nutrient-responsive properties and instead remains fixed across the genome. The resulting inflexibility in the face of changing nutritional conditions prevents taste plasticity and results in animals retaining the same sensory responses despite the different dietary environments. We show that OGT resides on the chromatin of the taste cells and that a subset of these loci are also diet-sensitive and also bound by PRC2.1. We further demonstrate that OGT and PRC2.1 orchestrate the responses of the taste cells to the high sugar environment by coordinating the expression of several homeobox transcription factors and their regulons. Finally, we report that parallel nutrient-dependent changes in gene expression occur in the lingual epithelium of rats exposed to high dietary sugar, and that a subset of these genes, particularly those encoding homeobox transcription factors, is decorated by OGT and PRC2; we also show convergence in biological processes altered by diet in the two animal models. Thus, we propose a model through which OGT and PRC2 function as a "food information" pathway that translates nutritional data into taste adaptations. More broadly, our findings reveal that the activity of metabolic enzymes plays an instructive role in neural and behavioral plasticity by guiding changes in chromatin accessibility in response to nutrient conditions.

\section{Results}

\section{The Nutrient Sensor OGT Directs Sensory Responses to Sweetness}

Similar to mammals, Drosophila melanogaster flies experience changes in sweet taste sensation when fed a diet supplemented with extra levels of sucrose, glucose, or fructose $(15-30 \%)^{27,30,31,34}$. In insects, the 60-80 sweet taste receptor-expressing neurons are located in the external and internal portions of the main taste organ, the proboscis (as well as in the legs), and send their projections to the Sub Esophageal Zone (SEZ) (Fig. 1A). Diet-induced taste plasticity results from lower electrophysiological ${ }^{27,31,34}$ and presynaptic calcium responses to sugar stimuli ${ }^{27,30,35}$. Our previous work found that the epigenetic repressor PRC2.1 regulates taste plasticity in response to the nutrient environment by controlling the expression of a transcriptional hub program important for the synaptic, signaling, and metabolic properties of these sensory neurons ${ }^{30}$. However, what signals or pathways guide the nutrient-dependent binding and reprogramming of the taste cells by PRC2.1 remains unknown.

Taste plasticity in flies occurs independently of fat accumulation, higher caloric intake, or extra sweetness, suggesting that sugar metabolism plays a role in this phenotype ${ }^{27}$; interestingly, diet also promotes aspects of mammalian taste plasticity independently of obesity ${ }^{21,29}$. In vertebrates and invertebrates, high energy intake, especially in the form of extra sugar, drives higher flux through the Hexosamine Biosynthesis Pathway (HBP), which results in higher concentrations of the signaling metabolite UDP-GIcNAc ${ }^{27,36-43}$. The nucleocytoplasmic enzyme OGT- which catalyzes the transfer of UDP-GlcNAc to proteins to change their 
function or stability- is sensitive to all cellular levels of this metabolite without substrate-inhibition, thus functioning as a bridge between the nutrient environment and cell physiology ${ }^{41,44-46}$. As such, OGT is considered a global nutrient sensor and has been implicated in pathological conditions linked to diet, such as heart disease and diabetes. The brain has among the highest levels of OGT expression and activity, and this enzyme is involved in different aspects of brain function, such as synaptic maturation and excitability, as well as brain processes such as development, energy balance, and neurodegeneration, reviewed in ${ }^{47}$. Thus, OGT could play an instructional role in taste plasticity by providing information about the nutritional context to the taste cells; if this were the case, we would expect that increasing the levels of OGT would mimic the effects of a high sugar diet, and result in lower sweet taste sensation ${ }^{41,44-46}$.

To test this hypothesis, we used the Gustatory receptor 5a GAL4 transgene (Gr5a-GAL4) ${ }^{48}$ to drive expression of $O G T$ in the sweet sensing neurons and measured sweet taste responses using the Proboscis Extension Assay (PER). In this behavioral assay, the labellum (Fig. 1A, left) is stimulated with low (5\%), medium (10\%), and high (30\%) concentrations of sucrose and the amount of proboscis extension is recorded $(0=$ no extension, 0.5 = partial extension, and 1 = full extension) (Fig. 1A, right). While control transgenic flies fed a control diet (CD) (Fig. 1B, gray) showed high PER to sucrose, Gr5a>OGT flies had PER comparable to that of animals on a sugar diet (Fig. 1B, green; compare to Fig. 1C, gray squares). Although the Gr5a-GAL4 transgene is not expressed during embryo or larval development, we used the temperature-sensitive tubulin-GAL80 ${ }^{\text {ts }}$ transgene to turn on the expression of OGT only in adult flies and control for potential developmental effects; these animals also showed sweet taste plasticity in the absence of diet (Fig. S1A). Further, we observed no changes in the number and gross morphology of the Gr5a+ neurons in the labellum when these were labeled with GFP, indicating that changes in taste are not due to cell loss (Fig. S1B). Thus, higher levels of OGT in the gustatory neurons promote changes in sweet taste comparable to those caused by a high sugar diet, opening the possibility that this enzyme may instruct taste plasticity in response to sugar levels.

To probe whether OGT is necessary for diet-induced taste plasticity, we tested the effects of knocking down this enzyme or inhibiting its catalytic activity on the PER of animals fed a control (CD) or high sugar diet (SD) for 7 days ${ }^{27,49}$. Knock-down of OGT (50\% efficiency ${ }^{27}$ ) in the Gr5a+ cells prevented the development of sweet taste plasticity in SD animals, as shown by the higher PER to sucrose of Gr5a>OGT $T^{R N A i}$ SD-flies compared to controls (Fig. S1B and C, which was also indistinguishable from those of CD flies, showing that OGT is necessary for taste plasticity in response to the dietary environment). The OGT C-terminal catalytic domain of OGT is required for its activity ${ }^{50,51}$, and mutations in this region decrease its output by $\sim 94-100 \%$ $\left(O G T^{H 537 A} \text { and } O G T^{K 872 M}\right)^{52}$. We thus asked whether the activity of OGT, and not just its levels, were required for diet-induced taste changes by testing the PER of flies carrying mutations in the catalytic domain. In contrast with OGT knockdown flies, $O G T^{H 537 A}$ and $O G T^{K 872 M}$ heterozygous flies (green) still showed a decrease in taste in response to the SD (Fig. 1C, these mutants are homozygous lethal, and thus tested as heterozygous, 
meaning $100 \%$ of OGT protein is present but only $~ 50 \%$ of its catalytic activity), suggesting that the activity of OGT is important for taste plasticity. To further validate this result, we supplemented control and sugar diets with OSMI-1, a small molecule inhibitor of OGT ${ }^{53}$. While animals on a sugar diet supplemented with vehicle (10\% dimethyl sulfoxide, DMSO) experienced lower sweet taste, those supplemented with OSMI-1 retained normal PER in a dose-dependent manner (Fig. S1D). Consistent with the role of OGT activity on taste plasticity, overexpression of OGT had no effect on sweet taste when OSMI was present (Fig. 1D). Together, these findings argue that the activity of OGT is necessary for diet-dependent sweet taste plasticity and that this metabolic enzyme plays a critical role in instructing changes in sweet taste in response to dietary conditions.

\section{OGT and PRC2.1 act in concert to tune sweet taste in response to diet}

A number of studies have reported that OGT interacts with chromatin and transcriptional regulators 44,46,54. In particular, OGT is known to genetically or physically interact with members of the Polycomb Group Proteins $(P c G)$ and play a role in PcG-mediated repression ${ }^{55-61}$. We previously identified the chromatin repressor PRC2.1 as a key player in D. melanogaster diet-induced taste plasticity. Although we showed that PRC2.1 regulated neural and behavioral taste plasticity in response to high dietary sugar by binding and modulating the expression of several transcription factors, ${ }^{30}$, we were unable to explain how this dietary-sensitivity arose. Since manipulations of OGT and PRC2.1 in the Gr5a+ neurons phenocopy each other, we hypothesized that OGT may provide the relevant nutrient context for PRC2.1; we thus asked whether OGT and PRC2.1 affect diet-induced taste plasticity by acting in concert or independently (Fig. 2A). To answer this question, we compared the PER of flies that overexpressed OGT in the Gr5a+cells and were also mutant for the PRC2.1 recruiter Polycomb like ( $\mathrm{Cl}$, essential to target the complex to chromatin), to flies that overexpressed OGT or were mutant for $P c l$ alone (Fig. 2A, purple arrow). OGT overexpression (Gr5a>OGT, green) promoted taste plasticity even in the absence of SD, while mutations in $\mathrm{PCl}\left(\mathrm{PCl}^{\mathrm{c429}}\right.$ pink) blocked it. In contrast, Gr5a>OGT; $P_{C} /^{c 429}$ flies failed to show any changes in taste in response to $S D$, compared to the control Gr5a-GAL4 flies (gray, circles vs. squares) (Fig. 2B, purple). Thus, OGT cannot induce sweet taste plasticity in the absence of a functional PRC2.1, placing this chromatin repressor genetically downstream of OGT. To probe whether the catalytic activity of PRC2.1 was required downstream of OGT, we tested the PER of Gr5a>OGT flies when the control diet was supplemented with the PRC2 inhibitor EEDi ${ }^{30,62}$, which blocks Histone 3 Lysine 27 methylation (H3K27m) by destabilizing the PRC2 complex (Fig. 2A). Consistent with the genetic epistasis data, we found that EEDi treatment completely blocked the ability of OGT to mimic the effects of SD and lower taste, as can be seen from the identical PER of control and experimental flies in the EEDi group compared to vehicle (Fig. 2C). Interestingly, we also found that the activity of OGT was also required for the ability of PRC2.1 to drive lower taste responses even in the absence of the high sugar environment (Fig. 2D), suggesting an interaction between the two players. Collectively, these experiments place OGT and PRC2.1 in the same genetic pathway that mediates diet-induced taste plasticity, and raise the possibility that 
OGT may provide the nutrient-dependent context for the action of PRC2.1 on the chromatin of the sweet sensing neurons.

\section{The nutrient-dependent chromatin redistribution of $P c /$ is contingent on OGT}

Previous work using DNA Adenine methyltransferase identification (Dam-ID or TaDA) showed that the occupancy of PRC2.1 on the chromatin of the Gr5a+ neurons changed at genomic loci that encoded for homeobox transcription factors involved in regulating processes such as synaptic function, neuron projection, signaling, differentiation, and metabolism ${ }^{30}$. Differential binding of PRC2.1 at these sites decreased chromatin accessibility and resulted in altered gene expression, lower calcium responses to sweetness, and lower taste in high-sugar diet fed flies ${ }^{30}$. The discovery that OGT and PRC2.1 act in concert to tune the responses of the sensory neurons depending on the dietary environment, raises the possibility that OGT may play an instructive role in the nutrient-dependent chromatin redistribution of PRC2.1. To test the hypothesis that diet-sensitive chromatin redistribution of PRC2.1 is dependent on the activity of OGT, we performed targeted Dam-IDin the presence of the OGT inhibitor OSMI-1 (Fig. 3A) ${ }^{63-65}$. To do this, we used the temperature sensitive tubulin-GAL80 ts to induce the expression of Dam::Pcl and Dam transgenes in the Gr5a+ sensory neurons in flies fed either a control or sugar diet supplemented with the OGT inhibitor OSMI-1 for 3 days (Fig. 3A); this time point was chosen to match the previous Dam ID experiments and based on the observation that taste plasticity develops within 3 days of exposure to SD ${ }^{27,30}$. Comparison of the Dam::Pc/ signal normalized to background Dam levels $\left(\log _{2}(\right.$ Dam::Pcl/Dam $\left.)\right)$ showed that while the CD+OSMI-1 and SD+OSMI-1 groups clustered based on diet, the variability between dietary conditions was less than $2 \%$ (Fig. S2A). Peak analysis for PCl occupancy revealed an 85\% overlap between the CD+OSMI-1 and SD+OSMI-1 (Fig. S2B). To identify how OSMI-1 influenced $P c l$ occupancy, we first compared the total number and the identity of the peaks of Dam::PCl in animals with or without OSMI-1 supplementation. The overall number $(\sim 1800)$ and identity $(80 \%)$ of peaks were identical (Fig. 3B and Fig. S2C), although 304 new peaks emerged in the Pcl+OSMI-1 condition (Fig. 3B). Pathway enrichment analysis revealed that most of the shared peaks were associated with gene regulatory GO terms such as development of anatomical structures, cell differentiation, neuron target attraction, and cell fate specification, while those unique to OSMI-1 treatment showed many metabolic GO terms, such as catecholamine and gluconate catabolic processes, and polyamine biosynthesis, (Fig. S2C and D). Together, this analysis revealed that OGT inhibition only had a small effect on the overall identity and number of peaks associated with PRC2.1, irrespective of diet.

We previously discovered that a high sugar diet environment redistributed the binding of PRC2.1 to the taste cells chromatin, with some loci showing higher (Fig. 3C, left Group 1) and others lower Pcl occupancy on SD (Fig. 3C, left Group 2) ${ }^{30}$. Thus, we reasoned that instead of affecting the number of loci bound by PRC2.1, OGT activity may instead influence the nutrient-dependent occupancy of $P c l$. To test this possibility, we compared the amount of $P C l$ signal in flies fed a CD and SD in the absence and presence of OSMI-1. Strikingly, 
we observed that treatment with OSMI-1 completely abolished the differential binding of Pcl between the CD and SD loci within both groups (Fig. 3C, right), suggesting that OGT activity is necessary for the nutrient-dependent chromatin redistribution of PRC2.1. Consistent with this, OSMI-1 also prevented the higher recruitment of Pcl to Polycomb Response Elements (PREs), the cis-regulatory sequences to which PcG proteins bind in D. melanogaster ${ }^{30}$ (Fig. 3D). Moreover, OSMI-1 treatment also blocked the SD-mediated decrease in CATaDa signal previously observed at Pcl peaks (Fig. 3E), a measure of chromatin accessibility that corresponds to DNAse I sensitive sites ${ }^{66}$ ). This indicates that the diet-dependent changes in PRC2.1 chromatin binding and accessibility depend on the activity of OGT, supporting the idea that OGT plays an instructive role in this process.

\section{OGT decorates the chromatin of the sweet taste neurons}

Our findings suggest that OGT guides changes in PRC2.1 chromatin occupancy and accessibility in response to the high sugar environment. OGT is a nucleoplasmic protein known to associate with DNA and chromatin binding factors such as Ten-Eleven Translocation Proteins and Host-Cell Factor 1 in the nucleus, as well as interacting with other nuclear proteins ${ }^{67-70}$. Although only one study showed this enzyme to reside on chromatin ${ }^{67}$, these observations are in line with the idea that metabolic enzymes "moonlight" in different cellular compartments to carry out alternative functions or interact with diverse partners ${ }^{71,72}$. We thus asked whether OGT may also be bound to the chromatin of the $\sim 60$ sweet taste neurons in the fly labellum. To test this possibility, we generated Dam::OGT (UAS-LT3-Dam::OGT) transgenic flies and measured the chromatin occupancy of Dam::OGT and Dam alone in the Gr5a+ neurons using TaDa after transiently inducing these transgenes for 18 hours (Fig. 4A) ${ }^{63,64}$. The normalized Dam::OGT replicates clustered together by diet (Fig. $\mathrm{S} 3 \mathrm{~A}$ ) and the chromatin accessibility profile of Dam at the Gr5a sweet taste receptor gene promoter was high, while at the bitter taste receptor promoter- expressed in adjacent cells-was low (Fig. S3B), suggesting that these new transgenes were appropriately targeted to the correct cell type.

Identification of Dam::OGT peaks revealed that OGT was indeed bound to the chromatin of the sweet taste cells, but that it targeted different types of loci, with $51 \%$ being introns and $\sim 30 \%$ Transcriptional Start Site (TSS) and promoters (Fig. 4B); of note, mammalian OGT is also largely associated with promoters and introns in embryonic stem cells ${ }^{67}$. OGT binding was enriched in the "yellow" transcriptionally active euchromatin type, characterized by H3K4 and H3K36 methylation in flies ${ }^{73}$ (Fig. S3C); consistent with this, we found that genes bound by OGT had higher expression levels in the sweet taste neurons compared to those not bound by it (Fig. 4C). However, a smaller portion of OGT was also enriched for the "blue" Polycomb heterochromatin decorated by H3K27 methylation and bound by PRC2 (Fig. S3C) ${ }^{30,73}$. Analysis of the OGT-bound peaks revealed approximately 1800-2000 intervals for each dietary condition (Fig. 4D). Although the majority of these intervals were shared between the two diets (Fig. 4D, 1392, find_peaks FDR <0.01), several hundreds loci showed differential binding and accessibility between the two dietary conditions (Fig. 4D and E). The decrease 
in chromatin accessibility in the SD condition was abolished with OSMI-1 treatment, suggesting that OGT activity plays a role in the openness of the sites it binds. Thus, OGT decorates the chromatin of the sweet taste cells, but only a portion of the OGT-bound sites were nutrient-sensitive in the tested dietary conditions; interestingly, most of these corresponded to loci found in "blue" PcG-bound chromatin.

To characterize the function of OGT targets, we performed pathway enrichment analysis using iPAGE ${ }^{74}$. On CD, OGT-decorated loci were involved in processes like signal transduction, membrane potential, and calmodulin-dependent protein kinase activity (Fig. 4F left); genes targeted by OGT in the SD condition were instead enriched in GPCR activity, synaptic target attraction, and transcription (Fig. 4F left). Comparison of the differentially bound Dam::OGTpeaks between the two diets, revealed an enrichment of regulatory/signaling and neural GO terms, including dendrite morphogenesis, neuron projection membrane, synaptic target attraction, neuron projection membrane, signal transduction, pattern formation and asymmetric cell division, as well as metabolic GO terms like triglyceride metabolic process (Fig. 4F right, for full iPAGE GO term analysis see Fig. S4), suggesting that these processes are nutrient-dependent and potentially regulated by OGT in the high sugar diet environment. In summary, our experiments show that OGT resides on the chromatin of the sweet taste cells; this chromatin corresponds to open and transcriptionally active domains, but its accessibility is sensitive to diet, and specifically decreased by SD in an OGT-dependent manner. Finally, we found that OGT-bound genes were strongly enriched in categories such as regulatory, signaling, development, and neural functions, but only a portion of them was responsive to changes in the dietary environment.

\section{OGT and PRC2.1 mark nutrient-sensitive chromatin in the sweet taste cells}

Since both OGT and PRC2.1 reside on the chromatin of the Gr5a+ sweet taste neurons, we asked whether there was an overlap in their occupancy. A comparison of the peaks occupied by Dam::Pcl with those bound by Dam::OGT, revealed a small number of shared intervals (Fig. 5A, 10\%). These 162 OGT x PCl peaks were enriched in the blue "Polycomb" chromatin ( $p<0.001$, permutation test), and consistent with this, the genes associated with these intervals had lower expression levels in the Gr5a+ neurons compared to those bound only by OGT (Fig. 5B, purple vs green), which are enriched for "yellow" chromatin (Fig. S3C) ${ }^{73}$. However, OGT x Pcl intervals had higher expression than those occupied by PRC2.1 alone, suggesting they may represent a subtype of Polycomb chromatin (Fig. 5B, purple vs pink). We next asked whether the dietary environment changed the occupancy of OGT and $\mathrm{Pcl}$ at these shared sites. This analysis showed that indeed more OGT and Pcl were bound to the shared peaks in the SD condition compared to CD (Fig. 5C); this magnitude was comparable or slightly larger than that at OGT or Pcl sites alone. It is also interesting to note that more OGT than Pcl was present at these sites in both diets (Fig. 5C). Strikingly, chromatin accessibility at shared OGT x Pcl was markedly decreased on SD compared to CD and this diet-dependent change was entirely dependent on OGT activity (Fig. 5D). Importantly, this nutrient-dependent shift in accessibility was 
3-fold higher at the shared loci compared to those bound by OGT or PRC2.1 alone (compared to Fig. 4E and $3 \mathrm{E})$, indicating that these sites may be characterized by a more open and diet-sensitive chromatin state.

To learn more about the biological functions of genes bound by both OGT and PRC2.1, we conducted GO term analysis using iPAGE (Fig. 5E). This revealed an enrichment in regulatory pathways involved in sequence specific DNA binding, regulation of cell proliferation, development, as well as neural processes such as Sodium channel regulator activity, activin receptor signaling, and dendrite development. In particular, we noticed that shared sites targeted Homeobox transcription factors we previously identified to be important for sweet taste function, specifically cad, Ptx1, and Scro (Fig. S5A) ${ }^{30}$. To assess the consequences of the diet-dependent shifts in PRC2.1 and OGT chromatin occupancy and accessibility, we isolated mRNAs associated with the ribosomes of the Gr5a+ cells using Translating mRNA Affinity Purification (TRAP) ${ }^{75}$ in flies fed a control or sugar diet supplemented with the OGT inhibitor OSMI-1 (Fig. S5B). Principal component analysis revealed that most of the variation between samples was due to diet (Fig. S5C); mRNAs specifically expressed in the Gr5a+ cells, such as the sweet taste receptor genes (Gr5a, Gr64f, and Gr64a) and the fatty acids taste receptor Ir56D, were enriched in the Gr5a+ fraction compared to the input, while bitter receptor genes (Gr66a and Gr32a) were depleted (Fig. S5D), showing that the selection of Gr5a+ mRNAs was successful and comparable to our prior experiments ${ }^{30}$. Differentially expressed genes (DEGs) showed a similar distribution in positive and negative changes (Fig. S5E), in contrast with the strong negative skew we previously observed in flies fed a sugar diet for 7 days, where $\sim 90 \%$ of genes had negative $12 \mathrm{fc}^{30}$. Specifically, we found that $52 \%$ of all the genes changed by SD ${ }^{30}$ were reverted (i.e., showed the opposite direction of change; $q<0.1$, Wald test) or unchanged (practical equivalence test using a null hypothesis of a change of at least 1.5 -fold and $\mathrm{q}<0.05$ ) by OSMI-1 treatment (Fig. 5F, gray are downregulated and red are upregulated). This argues that OGT activity is required for the changes in gene expression mediated by the high sugar diet environment. We previously found that this profound shift in mRNA levels in the sweet taste neurons of SD-flies required PRC2.1; we thus asked whether there was an overlap between the genes regulated, directly or indirectly, by OGT and PRC2.1. Indeed, mutations in $\mathrm{PCl}\left(\mathrm{PCl}^{\mathrm{c}^{429}}\right)$ had a similar effect to inhibition of OGT activity (Fig. 5F), including the reversal of homeobox transcription factors cad, Ptx1, and scro mRNA levels and those of their regulons (Fig. S5F) supporting the idea that OGT and PRC2.1 synergistically orchestrate responses to the dietary environment. Of note, a portion of PRC2.1-controlled genes were not dependent on OGT (specifically, the transcription factors GATAe, nub, and their regulons) and other genes changed by diet were not dependent on OGT nor PRC2 (such as cabut). Consistent with this hypothesis, genes affected by OSMI treatment were enriched in similar GO terms with those changed by SD via PRC2.1, including chemical synapses transmission, synaptic target attraction, neural projection membrane, signal transduction, regulation of asymmetric cell division, cell differentiation, and detection of chemical stimuli, as well as a number of metabolic processes (Fig. S6). Together, our data show that OGT and PRC2.1 co-occupy a small subset of genomic loci that are distinct from those bound by each protein alone. These loci are characterized by a more 
open chromatin, and are highly sensitive to the dietary environment; inhibition of OGT activity eliminates nutrient-dependent changes in chromatin accessibility, suggesting that the nutrient-responsiveness of these loci is conferred by OGT, likely by affecting PRC2.1 activity. Chromatin dynamics at these shared loci result in changes in taste by shifting the expression of transcriptional regulators, such as the homeobox genes Ptx 1 , cad, and scro, and their regulons; interfering with OGT or PRC2.1 function restores or prevents nutrient-dependent changes in mRNA levels, and blocks the development of taste plasticity.

\section{Transcriptional signatures of sugar diet exposure in the mammalian lingual epithelium}

Varying levels of sucrose in the diet also affect taste plasticity in mammals, reviewed recently in ${ }^{21,23,24}$. In particular, a recent study found that rats exposed to 30\% sucrose for 4-5 weeks presented lower responses of the chorda tympani nerve -a branch of the facial nerve that innervates the front $2 / 3$ of the tongue- to sweet stimuli ${ }^{32}$. Since this phenotype is strikingly similar to that observed in the sensory neurons of flies fed a $30 \%$ sucrose diet ${ }^{27,30,31,34}$, we asked whether nutrigenomics signatures parallel to those we observed in flies could be also detected in the mammalian tongue epithelia. To answer this question, we supplemented the diet of male rats with $30 \%$ liquid sucrose water for 5-6 weeks and then collected the anterior portion of the tongue epithelium for RNA sequencing (Fig. 6A). Rats were balanced for body weight at the start of the experiment and no changes were observed between groups following dietary treatment (419.25+/- 9.4 for water vs $440+/$ 15.8 grams for sucrose). The samples clustered by dietary condition (Fig. S7A) and exhibited high expression of epithelial markers and no or low expression of mesenchymal markers (Fig. S7B), showing that the isolation of the lingual epithelium was successful ${ }^{76,77}$. RNAseq analysis uncovered 2401 differentially expressed genes (DEGs) changed by diet, with 1673 up and 728 down (FDR< 0.1) (Fig. 6B). Pathway enrichment analysis revealed that these genes were involved in a multitude of biological processes, including positive and negative regulation of cellular process, regulation of developmental process, cell differentiation, cell death, regulation of signaling, regulation of metabolic process, as well as cellular responses to chemical stimuli (Fig. 6C). Thus, exposure to dietary sucrose in rats is also associated with changes in the levels of genes part of metabolic, signaling, and developmental processes. Of note, several of these GO terms were similar to those characterized in the DEGs of the labellum ${ }^{27}$ and sweet taste receptor cells ${ }^{30}$ of flies on a SD. We thus asked whether some of these diet-sensitive tongue DEGs may be associated with OGT and PRC2 as well. To do this we used published OGT chromatin immunoprecipitation data from mouse embryonic stem cells ${ }^{67}$ to compare the occupancy of this metabolic enzyme at the lingual DEGs vs. genes that did not show significant changes in expression. This in silico analysis revealed 1131 that were changed by sugar treatment in the tongue epithelium that were also decorated by OGT (Fig. 6D); interestingly, the occupancy of OGT at DEGs was higher than at non-DEGs (Fig. 6E), and the set of OGT-occupied genes was significantly enriched for DEGs $(p<0.001$, permutation test). Our findings in flies show that OGT and PRC2.1 mark a subset of diet-sensitive genes (Fig. 5); to probe whether there is evidence of a parallel phenomenon in mammals, we used chromatin immunoprecipitation data from MTF2 (the orthologue of $P c l$ ) ${ }^{78}$ and examined whether any of the 
OGT-decorated DEGs were also occupied by MTF2. This uncovered 192 DEGs occupied by both OGT and MTF2, which had modestly higher residence of MTF2 compared to non-DEGs (Fig. 6F). In fly sweet taste neurons, genes decorated by OGT and PRC2.1 were enriched for blue chromatin, the facultative heterochromatin characterized by binding of Polycomb Group Proteins and high H3K27 trimethylation (H3K27me3) in D. melanogaster ${ }^{73}$. We thus wondered whether the 192 OGT x MTF2 DEGs may also show features of Polycomb chromatin; indeed, we found significant enrichment in H3K27 methylation and depletion in H3K27 acetylation at these loci compared to non-DEGs (Fig. S7C and S7D; $p<0.0001$ in both cases, Mann-Whitney $U$ test on TSS-level differences) ${ }^{79}$. This finding was further validated by cross referencing these genes with the Epigenomics Roadmap ChIPseq and the Encode histone modification databases, which showed enrichment primarily in H3K27me3 modifications (q=9.75E-31 and 6.15E-11). To investigate the biological roles of these 192 DEGs, we conducted GO term analysis and found that they were involved in processes such as sequence-specific DNA binding, regulation of transcription, axon guidance by semaphorin-signaling pathway, regulation of protein kinase B signaling, regulation of p38 MAPK cascade, epithelial cell differentiation, neural crest migration, system development, and anatomical structure morphogenesis (Fig. S7E and Supplemental file). In humans, these DEGs are associated with an increased odd ratio for cancer and obesity (Jensen Diseases, odds ratio $=4.10$ and $7.77, q=0.013$ and 0.0079 respectively, ${ }^{80}$ ) and for waist-to-hip ratio (GWAS Catalogue 2019 , odds ratio=12.89, $\mathrm{q}=0.0255,{ }^{81}$ ), which is notable because there is a correlation between taste plasticity and metabolic disease ${ }^{21,82}$. Further, these sucrose DEGs also correspond to genes whose expression is altered by SARS-CoV-2 infection in vivo and in vitro in epithelial tissues (GSE47961:GPL6480:1, q= 0.02; GSE147507, q=0.005; GSE147507, q=0.005), which is interesting considering the COVID-19 causes chemosensory changes in humans ${ }^{83}$. We next investigated whether the OGT x MTF2 sucrose DEGs were expressed in subtypes of lingual epithelial cells by comparing them with genes known to be enriched in epithelial taste/progenitor cells or non-taste lingual epithelia in mice ${ }^{84-86}$. Strikingly, we found that $53 \%$ (102) of the sucrose DEGs corresponded to genes enriched in developing murine taste buds, including the transcription factors Pitx1 (the orthologue of the fly Ptx1, Fig. S5F), Jun, Ovol1, Gli3, Mafb, and Wnt and Notch signaling pathway components Wnt7b and Notch2; the other $47 \%$ of genes were enriched in non-taste supporting lingual cells, and of note, these included homeobox genes such as Pitx2, Hoxc13, DIx3, signaling factors part of the Notch pathway like Jag1, and axon targeting and neural projection signals like Sema 3f, 6a, and 7a (Fig. 6G and supplemental file). Together, these data suggest that exposure to high dietary sugar changes the expression of genes in the mammalian tongue epithelium that have important roles in taste bud development and taste function ${ }^{87,88}$ and indicate a potential role for OGT and PRC2.1 in these nutrigenomic signatures.

\section{Discussion}

OGT is an evolutionarily conserved enzyme thought to link the nutrient environment with cell physiology; here we deepened the understanding of this process by uncovering how OGT directs sensory 
plasticity to diet. Our studies have revealed that in sweet taste cells, OGT instructs neural adaptations to the dietary environment by guiding changes in chromatin accessibility. Specifically, we discovered that the activity of OGT is required for the redistribution of PRC2.1 occupancy, lower chromatin accessibility, and higher gene repression that occur with exposure to high dietary sugar (Fig. $6 \mathrm{H}$, summary). Our findings suggest that this was achieved via changes in the association of OGT with chromatin occupancy and the activity of PRC2.1. In particular, we uncovered that OGT and PRC2.1 share residence at 162 genomic loci and that these genes are more nutrient sensitive than those unoccupied by both proteins, as shown by the larger decrease in chromatin accessibility in response to the high sugar diet. Of note, the decreased accessibility at these shared loci was dependent on the activity of OGT, and interestingly more OGT and PRC2.1 resided at these loci in the high sugar environment (Fig. 5). These shared loci were characterized by an enrichment in transcription and regulatory factors involved in cell proliferation, differentiation, signaling, and neural activity. We further showed that these changes in chromatin accessibility drove the alterations in gene expression observed with the high sugar diet, because inhibition or mutations in OGT and PRC2.1 prevented or reversed them (Fig. 5F). We propose that this occurs by controlling the output of a transcriptional hub composed of homeobox transcription factors, Ptx1, Cad, and Scro (Fig. S5), which we previously showed are important for taste function ${ }^{30}$. Thus OGT and PRC2.1 are part of a cellular pathway integral for taste plasticity; in the absence of these two components, no neural adaptations develop and the cells remain largely cemented in the prior nutrient environment. Because nutrient sensing (OGT) and epigenetic (PRC2.1) components work in concert at the chromatin level to mediate transcriptional and cellular changes in response to the dietary environment (Fig. 2), we propose that OGT and PRC2 comprise a nutriepigenetic pathway that perceives and actualizes nutrient data into actions, specifically the action of sensory adaptations (Fig. 6H).

Our data in rats suggest that this nutriepigenetic mechanism may also be implicated in diet-induced taste plasticity in mammals. We found that exposure to $30 \%$ dietary sucrose for 5-6 weeks altered the expression of rat lingual epithelial genes involved in biological processes such as metabolism, cell death, differentiation, and signaling, and responses to chemical stimuli (Fig. 6A and B). Comparing these genes to the only available OGT ChIP data from ES cells, showed that this metabolic enzyme was enriched in the cis-regulatory regions of genes significantly changed by sucrose; moreover, we also observed a significant enrichment in binding at these gene by the Pcl orthologue MTF2 (Fig. 6E and F; $p<0.0001$ in both cases, Mann-Whitney $U$ test on TSS-level differences). In particular, we uncovered that of the genes changed by sucrose in the lingual epithelium, 192 were associated with both OGT and PRC2. These genes showed enrichment in the PRC2-histone modification H3K27me3, in a similar way to which fly shared OGT x PRC2.1 genes were enriched in blue Polycomb chromatin. Inspecting the identity of the shared OGT x MTF2 DEGs, revealed changes related to specific cellular processes in taste physiology, such as genes and pathways previously implicated in taste bud specification, renewal, and taste function, like Wnt, Notch, MAPK/p38, and BMP signaling, as well as transcription factors such as DIx3, Pitx1, Ovol1, Gli3, Hoxc13, and Jun ${ }^{84,85,88-94}$ (Fig. 
6G). The enrichment in differentially expressed taste function genes in those bound by OGT and MTF2, hints that this pathway may also regulate taste plasticity in mammals. To this end, it is particularly interesting to note the similarly in GO terms between the fly and rat OGT x PRC2 genes, in particular those related to DNA binding and transcriptional regulation, cell and anatomical structure development, differentiation, neural projection and synaptic/axon target attraction, and signaling. Among these, the homeobox transcription factor Ptx1 and its rat orthologues, Pitx1 and Pitx2, emerged as targets of sucrose decorated by OGT and PRC2 in both species (Fig. 5F and 6G). That said, evidence of the unique nature of fly (neural) and rat taste (epithelial) cells was also apparent, like the presence of more neural GO terms related to synaptic physiology in flies and more epithelial and cell division/differentiation terms in rats. Notably, some genes important for taste bud and taste cells differentiation, specification, and function, such as the transcription factors Hes1, as well as Wnt10b, Notch3, Fgf3 and a number of BMP members, like BMP6, BMP2k, BMP4, BMPr1a ${ }^{84,85,88-97}$, were also changed by sucrose but not part of the 192 shared loci. Although we don't know much about the role that Polycomb group proteins play in taste physiology in mammals, a recent study found that PRC1 is required for the proper maintenance of fungiform papilla in mice via regulation of Sonic HedgeHog signaling ${ }^{98}$. These genes and biological processes allude to the mechanisms through which taste plasticity may occur in these different species, likely by affecting synaptic plasticity and morphology in invertebrates, and taste bud renewal and taste cell to nerve communication in rodents. However, the enrichment in OGT and MTF2 binding and the presence of homeobox transcription factors and conserved signaling pathways in our data, highlights a convergence in the sensing and processing mechanisms involved in diet-induced taste plasticity in invertebrates and mammals. This convergence in biological processes with exposure to dietary sucrose may arise from the fact that neurons and epithelial cells share a common developmental origin, which may involve similar mechanisms to sense and respond to nutrient variation (discussed below). Thus, despite the anatomical differences, this nutriepigenetic pathway likely plays a conserved role in integrating and processing nutrient information into the physiological responses that underlie taste adaptations.

What are the implications of this interaction between the nutrient environment and sensory plasticity? In flies diet-induced taste plasticity causes obesity by inhibiting satiation ${ }^{27,35}$. In fact, correcting the activity of the sweet taste neurons or by preventing the chromatin and transcriptional remodeling by OGT and PRC2, protects animals from diet-induced obesity by restoring normal meal size ${ }^{27,30,35}$. Although the consequences of diet-induced taste plasticity in mammals are still not understood, the anticorrelation between taste function and $\mathrm{BMI}$, as well as the importance of sensory factors in satiation and satiety, suggest that this phenomenon could contribute to deregulated eating and obesity in humans ${ }^{23,82,99,100}$. Consistent with this idea and the fly data, we found that rat OGT x MTF2 DEGs were associated with obesity, waist-to-hip ratio, and cancer in humans. Together, our work highlights the role of OGT as a conserved nutrient-sensitive enzyme that translates messages about the dietary environment into sensory responses by cooperating with the epigenetic effector PRC2 (Fig. 6H). Work in invertebrates and accumulating evidence in mammals, shows that these sensory 
adaptations affect food intake and the risk for metabolic disease. This emphasizes the important role of nutriepigenetic signaling in physiology, health, and disease and raises a number of exciting questions and possibilities about the properties of this pathways in sensory plasticity, and of nutriepigenetic signaling more broadly.

First, our experiments suggest that OGT affects taste plasticity in response to diet by instructing changes in chromatin accessibility, specifically by promoting chromatin repression with a sugar diet. What are the mechanisms through which OGT accomplishes this? We found that PRC2.1 and its catalytic activity are required for OGT's effects. Further, PRC2.1 mutants block the transcriptional changes associated with diet-induced taste plasticity. Thus, PRC2.1 likely provides the repressive action that decreases chromatin accessibility and gene expression in the high sugar diet environment at the OGT x PRC2.1 genes. Although we did not measure cell-specific changes in H3K27me3 (which is not technically feasible), our experiments showing that inhibiting the complex's catalytic activity prevents OGT-mediated taste plasticity, suggest that H3K27me3 is the silencing signal. However, OGT could also affect chromatin accessibility by GlcNAcylating histones, although the function and effects of these histone modifications are still poorly understood ${ }^{41,70,101,102}$. Our data also showed that OGT catalytic activity is required for chromatin accessibility and gene repression, suggesting that OGT may affect PRC2. Consistent with this, several other studies have reported an interplay between OGT and Polycomb group proteins. For example, GlcNAcylation of PRC1-member Polyhomeotic is required for its repressive properties ${ }^{55,56}$. In mammalian cells, OGT influences stability and silencing activity of the PRC2 subunit Enhancer of Zeste Homologue 2 (Ezh2) -the enzyme that catalyzes H3K27 methylation; knockdown of OGT decreased H3K27 methylation and Ezh2 occupancy at promoters in cell culture, while OGT overexpression increased it ${ }^{57,60}$. OGT also affected the binding of Ezh2 to promoters of transcription factors in breast cancer cells, but not its stability ${ }^{103}$. Interestingly, depending on cell type and tissue, GlcNAcylation of Ezh2 did not affect its association with PRC2, but instead decreased enzymatic activity ${ }^{104}$ or stability ${ }^{105-107}$. Further, OGT-dependent recruitment of PRC2.1 to UNC5 family gene promoters led to their silencing in HCT116 colon cancer cells, which has been linked to the worsening of colorectal cancer by high carbohydrate diet in mammals ${ }^{61}$. In the hippocampus, knockdown of OGT reduced PRC2.1-mediated gene silencing during fear memory consolidation ${ }^{59}$. Thus, converging evidence suggests that OGT influences the function of Polycomb Group Proteins by either affecting their stability, binding to chromatin, or catalytic function, depending on the cell type. Due to the limitations of our system, our experiments do not address the physical mechanisms through which OGT instructs PRC2.1 in the taste cells. However, the finding that OGT activity is necessary for PRC2.1-mediated (Fig. 2) and diet-induced taste plasticity (Fig. 1), strongly suggests that OGT affects the repressive function of PRC2.1 in the sensory neurons.

The second question arises from our discovery that OGT occupies the chromatin of the sweet sensory neurons. To the best of our knowledge, only one other study showed that OGT occupies genomic intervals ${ }^{67}$, although this enzyme is known to be nuclear and cytoplasmic in both vertebrates and invertebrates ${ }^{45}$. Similar 
to results of Villa et al. (2013) in embryonic stem cells, we observed that OGT is present at different non-coding regions, including introns, promoters, enhancers, and TSS of genes. In the fly Gr5a+ neurons, these loci were enriched in neuronal and metabolic processes. Our analysis also revealed that in fly sweet taste neurons, OGT is generally associated with a type of euchromatin called "yellow", which includes housekeeping and actively elongating genes marked by H3K36me ${ }^{73}$. Interestingly, the Tudor domain of the mammalian orthologues of Pcl like MTF2 binds H3K36m to promote the intrusion of PRC2.1 into active genes and leads to their repression ${ }^{108-110}$. This could be a potential mechanism through which PRC2.1 is redistributed to actively transcribed loci in the sugar diet environment. Of note, the genes bound by both OGT and Pcl were characterized by a much higher CATaDa signal on CD and a much larger magnitude of change with SD than those bound by OGT or $\mathrm{Pcl}$ alone. This suggests that they may represent a type of chromatin that is particularly responsive to nutrients, similar to that observed in yeast, cancer cells, or plants ${ }^{111-113}$; we found similar transcriptionally active signals in the rat OGT x PRC2 DEGs (data not shown).

Finally, understanding more about the nature of nutrient-sensitive chromatin would help answer the question about specificity: what drives dynamics of nutrient-sensitive loci across different cell types and organisms? Indeed, we found that the OGT x PRC2 fly and rat loci were involved in similar biological processes and functions, such as DNA binding and transcriptional regulation, cell and anatomical structure development, differentiation, neural projection and synaptic/axon target attraction, and signaling. These two cells are obviously quite different, but they also share an ectodermal origin. We previously proposed that the outcome specificity that arises from cells experiencing the same nutrient perturbations, could result from which chromatin domains are actually accessible, which would be determined during development ${ }^{18,19}$. This mechanism would be particularly effective in neurons, where the modulation of developmental gene batteries has been postulated to affect synaptic plasticity ${ }^{30,114-116}$. This idea is supported by the observation that during development nutrients can direct the formation, not only of different cell types ${ }^{117,118}$, but of entirely different organisms, like worker and queen bees ${ }^{119,120}$. However, there must be more, because not all cells that share developmental origins or progenitors respond in similar ways. One possibility is that what makes accessible chromatin domains nutrient-sensitive is the association with "pools" of metabolic cofactors and/or of metabolic enzymes that "moonlight" in the nucleus. If that were the case, it is fascinating to speculate how these pools may be generated and maintained. In the case of OGT, our data show that only a portion of loci occupied by OGT show changes with diet, opening the possibility that OGT may move to different sites in the genome, or associate with different loci, depending on cellular signals. OGT is itself regulated by different nutrient and activity sensing kinases, such as adenosine monophosphate-activated protein kinase (AMPK), calcium/calmodulin-dependent protein kinase II (CaMKII), and insulin-regulated mitotic protein glycogen synthase kinase (GSK3; ${ }^{69,121}$. Thus, engagement of different signaling pathways could lead to cell-specific outcomes by promoting the interactions of OGT with unique complexes or by directing its subcellular localization depending on incoming signals (Fig. $6 \mathrm{H}$ ). Changes in the global or local levels of UDP-GIcNAc 
could be an additional level of regulation of OGT output, since the activity of this enzyme depends on the concentrations of this metabolite ${ }^{44,45}$. Thus, there could be a cell-specific code for OGT activation and outcome that depends on the environmental context the cell experiences and the particular association of OGT with PRC2 at unique chromatin domains; this may explain how fly and rat taste cells respond to high dietary sucrose with similar processes (leading to taste plasticity) despite the differences in anatomy and physiology. In the case of the taste cells in particular, calcium influx in response to the high sweetness of the diet together with higher GICNAc levels due to the higher sugar concentration, could lead to the interaction of OGT with particular protein complexes, like PRC2, or its recruitment to specific loci. In contrast, different triggers and effector mechanisms could be at play when OGT modulates synapse maturation, neural excitability, and responses in other circuits and environmental or activity conditions ${ }^{59,122-128}$. Of note, neural activity is known to increase GIcNAc levels, suggesting that one of the functions of OGT is to prepare the cell to face metabolic and redox stress ${ }^{47}$. Thus, we propose that OGT could provide the nutrient context (sensor) and PRC2.1 the outcome specificity (effector) for taste adaptations. In this proposed framework, the existence of cell-specific, nutrient-dependent chromatin loci and transcriptional responses may be shaped by both the permissive and prescriptive rules regulating OGT and its interaction with epigenetic effectors. Ultimately, this coordination would influence the processing of nutrient data into specific outcomes. In the current work, this coordination leads to diet-dependent transcriptional changes in the taste cells that contribute to taste plasticity by affecting synaptic plasticity in flies and, presumably, taste bud renewal or signal transduction in rats.

In conclusion, the combination of mechanisms acting through OGT could provide the cell and context specificity that transforms "nutritional data" - i.e. variations in nutrient and metabolite levels- into nutritional information ${ }^{129}$, as we proposed in the model shown in Fig. $6 \mathrm{H}$ (pink boxes). This information could then be processed and interpreted by gene regulatory processes such as those described here, to make "decisions" about how to respond to these environmental challenges and carry out physiological, neural, and behavioral changes. Importantly, the utilization of epigenetic mechanisms would also ensure a form of memory that would allow cells to react faster or more robustly to future alterations, while also potentially decreasing behavioral plasticity, as previously discussed ${ }^{30,130-132}$. Finally, since this mode of signaling requires the sensing of nutrient data, its contextualization into information, and its processing into action, it highlights the importance of interoceptive pathways in signal transduction. This may be particularly relevant for sensory cells, whose main role is to help the animal survive in complex and dangerous sensory landscapes and thus may benefit from using any type of information ${ }^{3}$. Thus, nutriepigenetic mechanisms are an important and newly recognized path for information flow in biological systems ${ }^{133-136}$ and have the advantage of amplifying transient, and often small, variations in nutrient and activity levels into robust reactions, which can be used to orchestrate responses to current and future environmental challenges. Here we uncovered how nutriepigenetic signaling plays a central role in translating information about the dietary environment into changes in neural and cell physiology that shape the animal sensory perception and the risk of obesity and metabolic disease. Future 
bioRxiv preprint doi: https://doi.org/10.1101/2021.12.17.473205; this version posted December 19, 2021. The copyright holder for this preprint (which was not certified by peer review) is the author/funder, who has granted bioRxiv a license to display the preprint in perpetuity. It is made available under aCC-BY-NC-ND 4.0 International license.

studies in this field will no doubt uncover fascinating insights about the rules of nutriepigenetic communication: these discoveries will illuminate the ways in which nutrition and gene expression converge to shape brain and sensory physiology and provide us with new tools to promote wellness and diminish the burden of disease. 


\section{METHODS}

\section{Fly husbandry, strains, and diets}

All flies were grown and fed cornmeal food (Bloomington Food $\mathrm{B}$ recipe) at $25^{\circ} \mathrm{C}$ and 45 to $55 \%$ humidity under a 12-hour light/12-hour dark cycle (Zeitgeber time 0 at 9:00 AM.), unless otherwise stated. Male flies were collected under $\mathrm{CO} 2$ anesthesia 1-3 days after eclosion and maintained in a vial that housed 35-40 flies. Flies were acclimated to their new vial environment for an additional 2 days and were moved to fresh food vials every other day.The GAL4/UAS system was used to express transgenes of interest using the Gustatory receptor $5 a$ Gr5a-GAL4 transgene. For each GAL4/UAS cross, transgenic controls were made by crossing the $w 1118^{c S}$ (gift from A. Simon, CS and w1118 lines from the Benzer laboratory) to GAL4 or UAS flies, sex-matched to those used in the GAL4/UAS cross. The fly lines used for this paper are listed in supplemental file 1.

For all dietary manipulations, the following compounds were mixed into standard cornmeal food (Bloomington Food $B$ recipe) ( 0.58 calories/g) by melting, mixing, and pouring new vials as in ${ }^{137}$ and ${ }^{49}$. For the $30 \%$ sugar

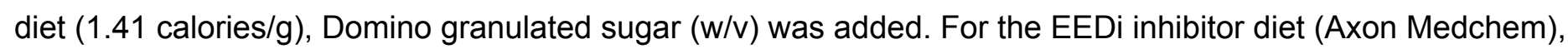
EEDi was prepared as in ${ }^{30}$. For the OSMI-1 inhibitor diet (Sigma Aldrich), OSMI-1 was solubilized in 10\%

DMSO and added to the control or $30 \%$ sugar diet at a total concentration of 10 or $30 \mu \mathrm{M}$.

\section{Rat dietary manipulations}

Male Sprague-Dawley rats obtained from Envigo (Indianapolis, IN) were approximately 65 days old upon arrival and singly housed in reverse light-dark conditions $(12 \mathrm{~h} / 12 \mathrm{~h})$. Rats were assigned to sucrose or control groups such that weight did not differ between conditions (water: 357.75 +/- $7.8 \mathrm{~g}$, sucrose: 360.75 +/- $8.8 \mathrm{~g}$ ) Both water (control) and sugar rats were given free access to standard chow (Lab Diet 5001: 4.07kcal/g; $13.5 \%$ fat, $28.5 \%$ protein, $58 \%$ carbohydrates; $\%$ of caloric content); sucrose rats were given a bottle of $30 \%$ sucrose diluted in tap water as their only liquid source, while water rats were given plain water alone. Food intake and body weight was monitored throughout. All procedures were approved by The University of Michigan Committee on the Use and Care of Animals in accordance with AAALAC and AVMA guidelines.

Rats in the water group consumed $23.78+/-0.49$ grams (mean+/-SEM) of chow/day, while sucrose rats ate $9.106+/-0.76$ grams of chow/day and $71.21+/-2.7$ grams of sucrose water/day. There was no significant difference in body weight between groups at the end of the study $(419.25+/-9.4$ for water vs $440+/-15.8$ grams for sucrose).

\section{Proboscis extension response}


Male flies were food-deprived for 18 to 20 hours in a vial with a Kimwipe dampened with Milli-Q filtered deionized water. Proboscis Extension Response was carried out as described in ${ }^{138}$. Scoring of extension responses were conducted manually, and whenever possible, experimenters were blinded.

\section{Proboscis immunofluorescence}

Probosces were dissected in 1× phosphate-buffered saline and fixed in 4\% paraformaldehyde, mounted in FocusClear (CelExplorer) on coverslips. Cell bodies were imaged using an FV1200 Olympus confocal with a $20 \times$ objective. Cells were counted using Imaris Image Analysis software.

\section{Tongue epithelia collection and RNA extraction}

Rats were humanely euthanized with an overdose of sodium pentobarbital (i.p.) prior to tissue collection. Tongue epithelium was isolated as previously described ${ }^{76,77}$. Briefly, the tongue was resected, washed in cold Tyrode's solution, then injected with a mixture of $1 \mathrm{mg} / \mathrm{ml}$ collagenase and $2 \mathrm{mg} / \mathrm{ml}$ dispase in Tyrode's solution and incubated for 30-45 minutes until the epithelium showed signs of bebbling (room temp). The anterior portion of the lingual epithelium, where the highest concentration of fungiform papillae are found, was then resected at the margins, peeled off the tongue, and immediately placed in Trizol reagent containing RNAlater (x volume). RNA extraction was performed per the manufacturer's instructions using the Qiagen RNeasy Micro kit (Cat. No. / ID: 74004) with on column DNAse digestion using the RNAse free DNAse set (Cat. No. / ID: 79254).

\section{Affinity purification of ribosome-associated mRNA (TRAP)}

Male fly heads (300 per replicate, $\sim 10,000$ Gr5a+ cells) were collected using sieves chilled in liquid nitrogen and on dry ice. Frozen tissue was then lysed as previously described ${ }^{30,75}$. From $10 \%$ of the total lysate, total RNA was extracted by TRIzol LS Reagent (Thermo Fisher Scientific, 10296010) to use as input. The remainder of the lysate was precleared by incubation with Dynabeads Protein G (Thermo Fisher Scientific, 10004D) for 2 hours and subsequently incubated with Dynabeads Protein G and an anti-Flag antibody (Sigma-Aldrich, F1804) at $4^{\circ} \mathrm{C}$ with rotation for 2 hours, then RNA was extracted from ribosomes bound to beads by TRIzol Reagent ${ }^{75}$.

\section{Targeted DNA adenine methyltransferase identification (TaDa) and Chromatin Accessibility TaDa} (CATada)

To generate the UAS-LT3-Dam::OGT construct, the coding region of the OGT gene was amplified from $w 1118^{C S}$ animals with the primers listed below and assembled into the UAS-LT3-DAM plasmid (gift from A. Brand, University of Cambridge) using the NEBuilder HiFi DNAAssembly kit based on the manufacturer's instructions (New England Biolabs). Transgenic animals were validated by reverse transcription PCR targeting the correct insert. The UAS-LT3-Dam::OGT and UAS-LT3-Dam line were crossed to the 
Gr5a-GAL4;tubulin-GAL80 8 . All animals were raised and maintained at $20^{\circ} \mathrm{C}$. Expression of Dam::OGT and Dam was induced at $28^{\circ} \mathrm{C}$ for 18 hours. For all experiments, 300 heads of males and female flies were collected per replicate on dry ice by sieving. DNA was extracted following kit instructions (Invitrogen). For identification of methylated regions, purified DNA was digested by Dpn I, followed by PCR purification of digested sequences. TaDa adaptors were ligated by T4 DNA ligase (NEB). Adapter ligated DNA was PCR-amplified and purified according to the protocol in ${ }^{63}$. Purified DNA was digested with Dpn II, followed by sonication to yield fragments averaging 300 base pairs. TaDa adaptors were removed from sonicated DNA by digestion followed by PCR purification and purified sonicated DNA was used for library preparation as in ${ }^{30}$ and 63 .

pUAST-Sxc.Forward gatctgGCCGGCGCaATGCATGTTGAACAAACACGAATAAATATG

pUAST-Sxc.Reverse gttccttcacaaagatcctTTATACTGCTGAAATGTGGTCCGGAAG

\section{Library preparation}

Generation of RNA sequencing (RNA-seq) libraries was with the Ovation SoLo RNA-seq System for Drosophila (NUGEN, 0502-96). All reactions included integrated Heat-Labile Double-Strand Specific DNase treatment (ArcticZymes, catalog no. 70800-201). The DNA-sequencing libraries for TaDa were generated using the Takara ThruPLEX Kit (catalog no. 022818). For rat RNA-seq, libraries were prepared using the Nugen Ovation Model organism (Rat \#0349-32) with 1/10th ERCC spike-in mix. These libraries were run on a NextSeq instrument using a HO 150 cycle cit (75x75 bp paired end reads). All Drosophila libraries were sequenced on the Illumina NextSeq platform (High-output kit v2 75 cycles) at the University of Michigan Genomics Core facility.

\section{High-throughput RNA-seq analysis}

Fastq files were assessed for quality using FastQC ${ }^{139}$. Reads with a quality score below 30 were discarded. Sequencing reads were aligned by STAR ${ }^{140}$ to dmel-all-chromosomes of the dm6 genome downloaded from Ensembl genomes. Counting was conducted by HTSeq ${ }^{141}$. Gene counts were used to call differential RNA abundance by DESeq2 ${ }^{142}$. Pipeline was generated from ${ }^{42}$. To determine the efficiency and cell specificity of the TRAP, pairwise comparisons were made between the Gr5a+-specific fraction and the input. For comparisons between dietary conditions, DESeq2 was only applied to the Gr5a+-specific IP condition. SD7 and $P c^{1429}$ datasets were analyzed from and described in ${ }^{30}$. A cutoff of $q<0.1$ was used to call DEGs. To identify overlap between datasets GeneOverlap was used ${ }^{143}$.

For rat RNAseq analysis all methods were the same as above except that reads were mapped to the Rnor_6.0 genome. DEGs are selected as genes with $q<0.1$ and non significant genes are $q>0.1$. Overlap between rat tongue epithelia DEGs with OGT and MTF2 was determined based on directly comparing the annotated genes 
associated with OGT peaks from ${ }^{67}$ and ${ }^{78}$ to rat epithelia DEGs with $\mathrm{q}<0.1$. For determining the non-DEGs used in Fig. 6C and D, we took a random set of genes (the same number as DEGs with OGT peaks) from the non-significant list and used it as a control for OGT and MTF2 occupancy. To intersect the intervals shared between OGT and MTF2, we identified the intervals of the shared OGT x MTF2 genes from the Mus musculus mm9 genome build and determined the overlapping intervals using Bedtools ${ }^{144}$. To identify the DEGs that share occupancy with both MTF2 and OGT and are also enriched in the taste epithelia, we compared our genes to the genes identified in ${ }^{84}$ that are characterized as enriched in the taste lingual epithelium. Data visualization was carried out in Rstudio using ggplot2 ${ }^{145}$ and the following packages: pheatmap ${ }^{146}$, Venneuler ${ }^{147}$, and EnhancedVolcano ${ }^{148}$. "Ward.D " clustering was used to cluster columns and rows in pheatmap.

\section{High-throughput TaDa and CATaDa analysis}

Fastq files were assessed for quality using FastQC ${ }^{139}$. Reads with a quality score below 30 were discarded. The damidseq_pipeline was used to align, extend, and generate log2 ratio files (Dam::Sxc/Dam) in GATC resolution as described previously ${ }^{149}$ and as in ${ }^{30}$. Reads were mapped by Bowtie $2{ }^{150}$ to dmel-all-chromosomes of the dm6 genome downloaded from Ensembl genomes, followed by read extension to $300 \mathrm{bp}$ (or to the closest GATC, whichever is first). Bam output is used to generate the ratio file in bedgraph format. Bedgraph files were converted to bigwig and visualized in the UCSC Genome Browser. Principal components analysis plots between biological replicates were computed by multibigwigSummary and plotCorrelation in deepTools ${ }^{151}$. Peaks were identified from ratio files using find_peaks (FDR <0.01) ${ }^{149}$ and as in ${ }^{30}$. Overlapping intervals or nearby intervals (up to 50bp) were merged into a single interval using mergeBed in BEDtools ${ }^{144}$. Intervals common in at least 2 replicate peak files were identified by Multiple Intersect in BEDtools and used to generate the consensus peaks ${ }^{144}$. For CATaDa experiments, all analyses were performed similar to those of TaDa with the exceptions thatDam only profiles were not normalized as ratios but shown as normalized binding profiles generated by converting bam files to bigwig files normalized to $1 \times \mathrm{dm} 6$ genome as reads per genome coverage (Sequencing depth is defined as the total number of mapped reads times the fragment length divided by the effective genome size). Binding intensity metaplots were made by computing a matrix for specified regions ${ }^{151}$. To determine the proportion of genes that fit within the various chromatin domain subtypes, we first matched Dam::OGT/Dam targets to coordinates identified by ${ }^{73}$ and then determined their gene count in each chromatin subtype (observed) compared to the whole genome (expected). Peak annotations were conducted using the HOMER annotatePeaks tool ${ }^{152}$ with the dm6 reference genome.

\section{Pathway enrichment analysis}

For all fly experiments GO term enrichment analysis was performed using the iPAGE package ${ }^{74}$, using gene-GO term associations extracted from the Flybase dmel 6.082015 _05 release. For all analyses iPAGE was run in discrete mode. Independence filtering was deactivated for all discrete calculations. All other iPAGE settings default values. All shown GO terms pass the significance tests for overall information described in ${ }^{74}$. 
For each term, bins that are outlined show especially strong contributions $[P$ value, such that a Benjamini-Hochberg FDR ${ }^{153}$ calculated across that row, yields $\left.q<0.05\right]$.

For rat experiments GO term analysis was performed using G:Profiler ${ }^{154}$ and Enrichr ${ }^{155-157}$ with the default functional profiling settings.

\section{Data analysis and statistics}

Statistical tests, sample size, and $P$ or $q$ values are listed in each figure legend. Kruskal-Wallis Dunn's multiple comparisons were used for all PER experiments. Comparisons are either to control diets within genotypes or transgenic controls within dietary conditions. All behavioral data were tested for normality and the appropriate statistical tests were applied if data were not normally distributed. For the RNA-seq expression datasets, we coupled our standard differential expression with a test for whether each gene could be flagged as "significantly not different" -- i.e., a gene for which we can confidently state that no substantial change in expression occurred (rather than just a lack of evidence for change, as would be inferred from a large $p$ value on the differential expression test). Defining a region of practical equivalence as a change of no more than 1.5-fold in either direction, we tested the null hypothesis of a change larger than 1.5-fold using the gene-wise estimates of the SE in log2fold change (reported by Deseq2) and the assumption that the actual I2fcs are normally distributed. Rejection of the null hypothesis is evidence that the gene's expression is not changed substantially between the conditions of interest. Python code for the practical equivalence test can be found on GitHub as calc_sig_unchanged.py. All data in the figures are shown as means \pm SEM, ${ }^{* * *} P<0.0001,{ }^{* * *} P<0.001,{ }^{* *} P$ $<0.01$, and ${ }^{*} P<0.05$, unless otherwise indicated.

\section{Data availability}

All high-throughput data are available at the GEO repository: GSE188757 for fly experiments and GSE191141 for rat experiments. For OGT ChIP-seq, data was obtained from GSE39154 ${ }^{67}$. For MTF2 occupancy analysis data was obtained from GSE $97805^{78}$.

Contributions: All experiments and data analysis were performed by AV with the exception of some of the iPAGE analysis and statistical tests, which was done by PF, and the rat handling and dissection, which were done by CRF. DW setup the RNAseq data analysis pipeline. MD and AV designed the project, interpreted the data, and wrote the manuscript, which was edited by all other authors. MD supervised the project and secured funding.

Acknowledgments: We thank the University of Indiana at Bloomington, the VDRC, the FLYORF stock collections, and all the investigators who shared fly lines with us. We thank Cameron W. Nobile and Christina May for feeding and weighting the rats during the dietary treatment and Dr. Charlotte Mistretta for advice on how to collect the tongue epithelium. We also thank Heather Bowling at Epistemic Al for providing access to 
the platform. Julia Kuhn designed some of the graphics for the manuscript. Funding: This work was funded by NIH R00 DK-97141 and NIH 1DP2DK-113750, the Klingenstein-Simons Fellowship in the Neurosciences, the Rita Allen Foundation and NSF CAREER 1941822 (to MD), the Rackham Predoctoral Fellowship (to AV), NIH R01DK106188 and 1R01DK115526 (to CRF), and NIH R35GM128637 (to PLF).

Conflict of interest: The authors declare no conflict of interest. 


\section{References}

1. Li, X., Egervari, G., Wang, Y., Berger, S.L., and Lu, Z. (2018). Regulation of chromatin and gene expression by metabolic enzymes and metabolites. Nat. Rev. Mol. Cell Biol. 19, 563-578.

2. Dai, Z., Ramesh, V., and Locasale, J.W. (2020). The evolving metabolic landscape of chromatin biology and epigenetics. Nat. Rev. Genet. 21, 737-753.

3. Dusenbery, D.B. (1996). Information Is Where You Find It. Biol. Bull. 191, 124-128.

4. Yucel, N., Wang, Y.X., Mai, T., Porpiglia, E., Lund, P.J., Markov, G., Garcia, B.A., Bendall, S.C., Angelo, M., and Blau, H.M. (2019). Glucose Metabolism Drives Histone Acetylation Landscape Transitions that Dictate Muscle Stem Cell Function. Cell Rep. 27, 3939-3955.e6.

5. Folmes, C.D.L., Nelson, T.J., Martinez-Fernandez, A., Arrell, D.K., Lindor, J.Z., Dzeja, P.P., Ikeda, Y., Perez-Terzic, C., and Terzic, A. (2011). Somatic oxidative bioenergetics transitions into pluripotency-dependent glycolysis to facilitate nuclear reprogramming. Cell Metab. 14, 264-271.

6. Takubo, K., Nagamatsu, G., Kobayashi, C.I., Nakamura-Ishizu, A., Kobayashi, H., Ikeda, E., Goda, N., Rahimi, Y., Johnson, R.S., Soga, T., et al. (2013). Regulation of glycolysis by Pdk functions as a metabolic checkpoint for cell cycle quiescence in hematopoietic stem cells. Cell Stem Cell 12, 49-61.

7. Oburoglu, L., Tardito, S., Fritz, V., de Barros, S.C., Merida, P., Craveiro, M., Mamede, J., Cretenet, G., Mongellaz, C., An, X., et al. (2014). Glucose and glutamine metabolism regulate human hematopoietic stem cell lineage specification. Cell Stem Cell 15, 169-184.

8. Qiu, J., Villa, M., Sanin, D.E., Buck, M.D., O'Sullivan, D., Ching, R., Matsushita, M., Grzes, K.M., Winkler, F., Chang, C.-H., et al. (2019). Acetate Promotes T Cell Effector Function during Glucose Restriction. Cell Rep. 27, 2063-2074.e5.

9. Liu, P.-S., Wang, H., Li, X., Chao, T., Teav, T., Christen, S., Di Conza, G., Cheng, W.-C., Chou, C.-H., Vavakova, M., et al. (2017). a-ketoglutarate orchestrates macrophage activation through metabolic and epigenetic reprogramming. Nat. Immunol. 18, 985-994.

10. Yu, W., Wang, Z., Zhang, K., Chi, Z., Xu, T., Jiang, D., Chen, S., Li, W., Yang, X., Zhang, X., et al. (2019). One-Carbon Metabolism Supports S-Adenosylmethionine and Histone Methylation to Drive Inflammatory Macrophages. Mol. Cell 75, 1147-1160.e5.

11. Gao, X., Sanderson, S.M., Dai, Z., Reid, M.A., Cooper, D.E., Lu, M., Richie, J.P., Jr, Ciccarella, A., Calcagnotto, A., Mikhael, P.G., et al. (2019). Dietary methionine influences therapy in mouse cancer models and alters human metabolism. Nature 572, 397-401.

12. Wang, Z., Yip, L.Y., Lee, J.H.J., Wu, Z., Chew, H.Y., Chong, P.K.W., Teo, C.C., Ang, H.Y.-K., Peh, K.L.E., Yuan, J., et al. (2019). Methionine is a metabolic dependency of tumor-initiating cells. Nat. Med. 25, 825-837.

13. Guo, X., Kimura, A., Azuchi, Y., Akiyama, G., Noro, T., Harada, C., Namekata, K., and Harada, T. (2016). Caloric restriction promotes cell survival in a mouse model of normal tension glaucoma. Sci. Rep. 6 , 33950.

14. Morris, J.P., 4th, Yashinskie, J.J., Koche, R., Chandwani, R., Tian, S., Chen, C.-C., Baslan, T., Marinkovic, Z.S., Sánchez-Rivera, F.J., Leach, S.D., et al. (2019). a-Ketoglutarate links p53 to cell fate during tumour suppression. Nature 573, 595-599.

15. Roberts, M.N., Wallace, M.A., Tomilov, A.A., Zhou, Z., Marcotte, G.R., Tran, D., Perez, G., 
Gutierrez-Casado, E., Koike, S., Knotts, T.A., et al. (2018). A Ketogenic Diet Extends Longevity and Healthspan in Adult Mice. Cell Metab. 27, 1156.

16. Newman, J.C., Covarrubias, A.J., Zhao, M., Yu, X., Gut, P., Ng, C.-P., Huang, Y., Haldar, S., and Verdin, E. (2017). Ketogenic Diet Reduces Midlife Mortality and Improves Memory in Aging Mice. Cell Metab. 26, 547-557.e8.

17. Papsdorf, K., and Brunet, A. (2019). Linking Lipid Metabolism to Chromatin Regulation in Aging. Trends Cell Biol. 29, 97-116.

18. Vaziri, A., and Dus, M. (2021). Brain on food: The neuroepigenetics of nutrition. Neurochem. Int. 149, 105099.

19. Sarangi, M., and Dus, M. (2021). Crème de la Créature: Dietary Influences on Behavior in Animal Models. Frontiers in Behavioral Neuroscience; Lausanne.

20. Egervari, G., Glastad, K.M., and Berger, S.L. (2020). Food for thought. Science 370, 660-662.

21. May, C.E., and Dus, M. (2021). Confection Confusion: Interplay Between Diet, Taste, and Nutrition. Trends in Endocrinology \& Metabolism 32, 95-105.

22. Keast, R.S.J. (2016). Effects of sugar and fat consumption on sweet and fat taste. Current Opinion in Behavioral Sciences 9, 55-60.

23. Reed, D.R., Alhadeff, A.L., Beauchamp, G.K., Chaudhari, N., Duffy, V.B., Dus, M., Fontanini, A., Glendinning, J.I., Green, B.G., Joseph, P.V., et al. (2020). NIH Workshop Report: sensory nutrition and disease. Am. J. Clin. Nutr.

24. Harnischfeger, F., and Dando, R. (2021). Obesity-induced taste dysfunction, and its implications for dietary intake. Int. J. Obes. 45, 1644-1655.

25. Maliphol, A.B., Garth, D.J., and Medler, K.F. (2013). Diet-induced obesity reduces the responsiveness of the peripheral taste receptor cells. PLoS One 8, e79403.

26. Chen, K., Yan, J., Suo, Y., Li, J., Wang, Q., and Lv, B. (2010). Nutritional status alters saccharin intake and sweet receptor mRNA expression in rat taste buds. Brain Res. 1325, 53-62.

27. May, C.E., Vaziri, A., Lin, Y.Q., Grushko, O., Khabiri, M., Wang, Q.-P., Holme, K.J., Pletcher, S.D., Freddolino, P.L., Neely, G.G., et al. (2019). High Dietary Sugar Reshapes Sweet Taste to Promote Feeding Behavior in Drosophila melanogaster. Cell Rep. 27, 1675-1685.e7.

28. Weiss, M.S., Hajnal, A., Czaja, K., and Di Lorenzo, P.M. (2019). Taste Responses in the Nucleus of the Solitary Tract of Awake Obese Rats Are Blunted Compared With Those in Lean Rats. Front. Integr. Neurosci. 13, 35.

29. Ahart, Z.C., Martin, L.E., Kemp, B.R., Banik, D.D., Roberts, S.G.E., Torregrossa, A.M., and Medler, K. (2019). Differential effects of diet and weight on taste responses in diet-induced obese mice. bioRxiv, 564211.

30. Vaziri, A., Khabiri, M., Genaw, B.T., May, C.E., Freddolino, P.L., and Dus, M. (2020). Persistent epigenetic reprogramming of sweet taste by diet. Sci Adv 6.

31. Wang, Q.-P., Lin, Y.Q., Lai, M.-L., Su, Z., Oyston, L.J., Clark, T., Park, S.J., Khuong, T.M., Lau, M.-T., Shenton, V., et al. (2020). PGC1a Controls Sucrose Taste Sensitization in Drosophila. Cell Rep. 31, 107480. 
32. McCluskey, L.P., He, L., Dong, G., and Harris, R. (2020). Chronic exposure to liquid sucrose and dry sucrose diet have differential effects on peripheral taste responses in female rats. Appetite 145, 104499.

33. Shahbandi, A., Choo, E., and Dando, R. (2018). Receptor Regulation in Taste: Can Diet Influence How We Perceive Foods? J 1, 106-115.

34. Ganguly, A., Dey, M., Scott, C., Duong, V.-K., and Dahanukar, A. (2021). Dietary Macronutrient Imbalances Lead to Compensatory Changes in Peripheral Taste via Independent Signaling Pathways. J. Neurosci.

35. May, C.E., Rosander, J., Gottfried, J., Dennis, E., and Dus, M. (2020). Dietary sugar inhibits satiation by decreasing the central processing of sweet taste. Elife 9.

36. Hanover, J.A., Krause, M.W., and Love, D.C. (2010). The hexosamine signaling pathway: O-GlcNAc cycling in feast or famine. Biochim. Biophys. Acta 1800, 80-95.

37. Bouché, C., Serdy, S., Kahn, C.R., and Goldfine, A.B. (2004). The cellular fate of glucose and its relevance in type 2 diabetes. Endocr. Rev. 25, 807-830.

38. Hawkins, M., Barzilai, N., Liu, R., Hu, M., Chen, W., and Rossetti, L. (1997). Role of the glucosamine pathway in fat-induced insulin resistance. J. Clin. Invest. 99, 2173-2182.

39. Marshall, S., Nadeau, O., and Yamasaki, K. (2004). Dynamic actions of glucose and glucosamine on hexosamine biosynthesis in isolated adipocytes: differential effects on glucosamine 6-phosphate, UDP-N-acetylglucosamine, and ATP levels. J. Biol. Chem. 279, 35313-35319.

40. Wang, J., Liu, R., Hawkins, M., Barzilai, N., and Rossetti, L. (1998). A nutrient-sensing pathway regulates leptin gene expression in muscle and fat. Nature 393, 684-688.

41. Olivier-Van Stichelen, S., Wang, P., Comly, M., Love, D.C., and Hanover, J.A. (2017). Nutrient-driven O-linked $\mathrm{N}$-acetylglucosamine (O-GlcNAc) cycling impacts neurodevelopmental timing and metabolism. J. Biol. Chem. 292, 6076-6085.

42. Wilinski, D., Winzeler, J., Duren, W., Persons, J.L., Holme, K.J., Mosquera, J., Khabiri, M., Kinchen, J.M., Freddolino, P.L., Karnovsky, A., et al. (2019). Rapid metabolic shifts occur during the transition between hunger and satiety in Drosophila melanogaster. Nat. Commun. 10, 4052.

43. Na, J., Sweetwyne, M.T., Park, A.S.D., Susztak, K., and Cagan, R.L. (2015). Diet-Induced Podocyte Dysfunction in Drosophila and Mammals. Cell Rep. 12, 636-647.

44. Olivier-Van Stichelen, S., and Hanover, J.A. (2015). You are what you eat: O-linked N-acetylglucosamine in disease, development and epigenetics. Curr. Opin. Clin. Nutr. Metab. Care 18, 339-345.

45. Hart, G.W. (2019). Nutrient regulation of signaling and transcription. J. Biol. Chem. 294, 2211-2231.

46. Hardivillé, S., and Hart, G.W. (2014). Nutrient regulation of signaling, transcription, and cell physiology by O-GIcNAcylation. Cell Metab. 20, 208-213.

47. Lagerlöf, O. (2018). O-GIcNAc cycling in the developing, adult and geriatric brain. J. Bioenerg. Biomembr. $50,241-261$.

48. Chyb, S., Dahanukar, A., Wickens, A., and Carlson, J.R. (2003). Drosophila Gr5a encodes a taste receptor tuned to trehalose. Proc. Natl. Acad. Sci. U. S. A. 100 Supp/ 2, 14526-14530.

49. Na, J., Musselman, L.P., Pendse, J., Baranski, T.J., Bodmer, R., Ocorr, K., and Cagan, R. (2013). A Drosophila Model of High Sugar Diet-Induced Cardiomyopathy. PLoS Genetics 9, e1003175. 
50. Kreppel, L.K., Blomberg, M.A., and Hart, G.W. (1997). Dynamic glycosylation of nuclear and cytosolic proteins. Cloning and characterization of a unique O-GIcNAc transferase with multiple tetratricopeptide repeats. J. Biol. Chem. 272, 9308-9315.

51. Lubas, W.A., and Hanover, J.A. (2000). Functional expression of O-linked GlcNAc transferase. Domain structure and substrate specificity. J. Biol. Chem. 275, 10983-10988.

52. Mariappa, D., Zheng, X., Schimpl, M., Raimi, O., Ferenbach, A.T., Müller, H.-A.J., and van Aalten, D.M.F. (2015). Dual functionality of O-GlcNAc transferase is required for Drosophila development. Open Biol. 5, 150234.

53. Trapannone, R., Rafie, K., and van Aalten, D.M.F. (2016). O-GlcNAc transferase inhibitors: current tools and future challenges. Biochem. Soc. Trans. 44, 88-93.

54. Decourcelle, A., Leprince, D., and Dehennaut, V. (2019). Regulation of Polycomb Repression by O-GIcNAcylation: Linking Nutrition to Epigenetic Reprogramming in Embryonic Development and Cancer. Front. Endocrinol. 10, 117.

55. Gambetta, M.C., Oktaba, K., and Müller, J. (2009). Essential role of the glycosyltransferase sxc/Ogt in polycomb repression. Science 325, 93-96.

56. Gambetta, M.C., and Müller, J. (2014). O-GlcNAcylation prevents aggregation of the Polycomb group repressor polyhomeotic. Dev. Cell 31, 629-639.

57. Chu, C.-S., Lo, P.-W., Yeh, Y.-H., Hsu, P.-H., Peng, S.-H., Teng, Y.-C., Kang, M.-L., Wong, C.-H., and Juan, L.-J. (2014). O-GlcNAcylation regulates EZH2 protein stability and function. Proc. Natl. Acad. Sci. U. S. A. $111,1355-1360$.

58. Maury, J.J.P., El Farran, C.A., Ng, D., Loh, Y.-H., Bi, X., Bardor, M., and Choo, A.B.-H. (2015). RING1B O-GlcNAcylation regulates gene targeting of polycomb repressive complex 1 in human embryonic stem cells. Stem Cell Res. 15, 182-189.

59. Butler, A.A., Sanchez, R.G., Jarome, T.J., Webb, W.M., and Lubin, F.D. (2019). O-GlcNAc and $\mathrm{EZH} 2-$ mediated epigenetic regulation of gene expression during consolidation of fear memories. Learn. Mem. 26, 373-379.

60. Sakabe, K., and Hart, G.W. (2010). O-GlcNAc Transferase Regulates Mitotic Chromatin Dynamics*. J. Biol. Chem. 285, 34460-34468.

61. Decourcelle, A., Very, N., Djouina, M., Loison, I., Thévenet, J., Body-Malapel, M., Lelièvre, E., Coqueret, O., Leprince, D., El Yazidi-Belkoura, I., et al. (2020). O-GlcNAcylation Links Nutrition to the Epigenetic Downregulation of UNC5A during Colon Carcinogenesis. Cancers 12.

62. Qi, W., Zhao, K., Gu, J., Huang, Y., Wang, Y., Zhang, H., Zhang, M., Zhang, J., Yu, Z., Li, L., et al. (2017). An allosteric PRC2 inhibitor targeting the H3K27me3 binding pocket of EED. Nat. Chem. Biol. 13, 381-388.

63. Marshall, O.J., Southall, T.D., Cheetham, S.W., and Brand, A.H. (2016). Cell-type-specific profiling of protein-DNA interactions without cell isolation using targeted DamID with next-generation sequencing. Nat. Protoc. 11, 1586-1598.

64. van Steensel, B., and Henikoff, S. (2000). Identification of in vivo DNA targets of chromatin proteins using tethered dam methyltransferase. Nat. Biotechnol. 18, 424-428.

65. Aughey, G.N., Gomez, A.E., Thomson, J., Yin, H., and Southhall, T.D. (2018). CATaDa reveals global 
bioRxiv preprint doi: https://doi.org/10.1101/2021.12.17.473205; this version posted December 19, 2021. The copyright holder for this preprint (which was not certified by peer review) is the author/funder, who has granted bioRxiv a license to display the preprint in perpetuity. It is made available under aCC-BY-NC-ND 4.0 International license.

remodelling of chromatin accessibility during stem cell differentiation in vivo. eLife, 7.

66. Sen, S.Q., Chanchani, S., Southall, T.D., and Doe, C.Q. (2019). Neuroblast-specific open chromatin allows the temporal transcription factor, Hunchback, to bind neuroblast-specific loci. Elife 8.

67. Vella, P., Scelfo, A., Jammula, S., Chiacchiera, F., Williams, K., Cuomo, A., Roberto, A., Christensen, J., Bonaldi, T., Helin, K., et al. (2013). Tet proteins connect the O-linked N-acetylglucosamine transferase Ogt to chromatin in embryonic stem cells. Mol. Cell 49, 645-656.

68. Gao, J., Yang, Y., Qiu, R., Zhang, K., Teng, X., Liu, R., and Wang, Y. (2018). Proteomic analysis of the OGT interactome: novel links to epithelial-mesenchymal transition and metastasis of cervical cancer. Carcinogenesis 39, 1222-1234.

69. Hart, G.W., Slawson, C., Ramirez-Correa, G., and Lagerlof, O. (2011). Cross talk between O-GlcNAcylation and phosphorylation: roles in signaling, transcription, and chronic disease. Annu. Rev. Biochem. 80, 825-858.

70. Gambetta, M.C., and Müller, J. (2015). A critical perspective of the diverse roles of O-GlcNAc transferase in chromatin. Chromosoma 124, 429-442.

71. Boukouris, A.E., Zervopoulos, S.D., and Michelakis, E.D. (2016). Metabolic Enzymes Moonlighting in the Nucleus: Metabolic Regulation of Gene Transcription. Trends Biochem. Sci. 41, 712-730.

72. Xu, D., Shao, F., Bian, X., Meng, Y., Liang, T., and Lu, Z. (2021). The Evolving Landscape of Noncanonical Functions of Metabolic Enzymes in Cancer and Other Pathologies. Cell Metab. 33, 33-50.

73. Filion, G.J., van Bemmel, J.G., Braunschweig, U., Talhout, W., Kind, J., Ward, L.D., Brugman, W., de Castro, I.J., Kerkhoven, R.M., Bussemaker, H.J., et al. (2010). Systematic protein location mapping reveals five principal chromatin types in Drosophila cells. Cell 143, 212-224.

74. Goodarzi, H., Elemento, O., and Tavazoie, S. (2009). Revealing global regulatory perturbations across human cancers. Mol. Cell 36, 900-911.

75. Chen, X., and Dickman, D. (2017). Development of a tissue-specific ribosome profiling approach in Drosophila enables genome-wide evaluation of translational adaptations. PLoS Genet. 13, e1007117.

76. Meisel, C.T., Pagella, P., Porcheri, C., and Mitsiadis, T.A. (2020). Three-Dimensional Imaging and Gene Expression Analysis Upon Enzymatic Isolation of the Tongue Epithelium. Front. Physiol. 11, 825.

77. Venkatesan, N., Boggs, K., and Liu, H.-X. (2016). Taste Bud Labeling in Whole Tongue Epithelial Sheet in Adult Mice. Tissue Eng. Part C Methods 22, 332-337.

78. Li, H., Liefke, R., Jiang, J., Kurland, J.V., Tian, W., Deng, P., Zhang, W., He, Q., Patel, D.J., Bulyk, M.L., et al. (2017). Polycomb-like proteins link the PRC2 complex to CpG islands. Nature 549, 287-291.

79. Banaszynski, L.A., Wen, D., Dewell, S., Whitcomb, S.J., Lin, M., Diaz, N., Elsässer, S.J., Chapgier, A., Goldberg, A.D., Canaani, E., et al. (2013). Hira-Dependent Histone H3.3 Deposition Facilitates PRC2 Recruitment at Developmental Loci in ES Cells. Cell 155, 107-120.

80. DISEASES https://diseases.jensenlab.org/Search.

81. Burdett, T., Hastings, E., Welter, D., SPOT, EMBL-EBI, and NHGRI GWAS Catalog. https://www.ebi.ac.uk/gwas/.

82. Bartoshuk, L.M., Duffy, V.B., Hayes, J.E., Moskowitz, H.R., and Snyder, D.J. (2006). Psychophysics of sweet and fat perception in obesity: problems, solutions and new perspectives. Philos. Trans. R. Soc. 
Lond. B Biol. Sci. 361, 1137-1148.

83. Cooper, K.W., Brann, D.H., Farruggia, M.C., Bhutani, S., Pellegrino, R., Tsukahara, T., Weinreb, C., Joseph, P.V., Larson, E.D., Parma, V., et al. (2020). COVID-19 and the Chemical Senses: Supporting Players Take Center Stage. Neuron 107, 219-233.

84. Golden, E.J., Larson, E.D., Shechtman, L.A., Trahan, G.D., Gaillard, D., Fellin, T.J., Scott, J.K., Jones, K.L., and Barlow, L.A. (2021). Onset of taste bud cell renewal starts at birth and coincides with a shift in SHH function. Elife 10.

85. Qin, Y., Sukumaran, S.K., Jyotaki, M., Redding, K., Jiang, P., and Margolskee, R.F. (2018). Gli3 is a negative regulator of Tas1r3-expressing taste cells. PLoS Genet. 14, e1007058.

86. Hevezi, P., Moyer, B.D., Lu, M., Gao, N., White, E., Echeverri, F., Kalabat, D., Soto, H., Laita, B., Li, C., et al. (2009). Genome-wide analysis of gene expression in primate taste buds reveals links to diverse processes. PLoS One 4, e6395.

87. Kapsimali, M., and Barlow, L.A. (2013). Developing a sense of taste. Semin. Cell Dev. Biol. 24, 200-209.

88. Barlow, L.A., and Klein, O.D. (2015). Developing and regenerating a sense of taste. Curr. Top. Dev. Biol. $111,401-419$.

89. Fan, D., Chettouh, Z., Consalez, G.G., and Brunet, J.-F. (2019). Taste bud formation depends on taste nerves. Elife 8.

90. Rasch, L.J., Martin, K.J., Cooper, R.L., Metscher, B.D., Underwood, C.J., and Fraser, G.J. (2016). An ancient dental gene set governs development and continuous regeneration of teeth in sharks. Dev. Biol. 415, 347-370.

91. Lamba, P., Hjalt, T.A., and Bernard, D.J. (2008). Novel forms of Paired-like homeodomain transcription factor 2 (PITX2): generation by alternative translation initiation and mRNA splicing. BMC Mol. Biol. 9, 31.

92. Godwin, A.R., and Capecchi, M.R. (1998). Hoxc13 mutant mice lack external hair. Genes Dev. 12, 11-20.

93. Shandilya, J., Gao, Y., Nayak, T.K., Roberts, S.G.E., and Medler, K.F. (2016). AP1 transcription factors are required to maintain the peripheral taste system. Cell Death Dis. 7, e2433.

94. Ota, M.S., Kaneko, Y., Kondo, K., Ogishima, S., Tanaka, H., Eto, K., and Kondo, T. (2009). Combined in silico and in vivo analyses reveal role of Hes1 in taste cell differentiation. PLoS Genet. 5, e1000443.

95. Nishiguchi, Y., Ohmoto, M., Koki, J., Enomoto, T., Kominami, R., Matsumoto, I., and Hirota, J. (2016). Bcl11b/Ctip2 is required for development of lingual papillae in mice. Dev. Biol. 416, 98-110.

96. Behrens, M., Bartelt, J., Reichling, C., Winnig, M., Kuhn, C., and Meyerhof, W. (2006). Members of RTP and REEP Gene Families Influence Functional Bitter Taste Receptor Expression*. J. Biol. Chem. 281, 20650-20659.

97. Zhou, Y., Liu, H.-X., and Mistretta, C.M. (2006). Bone morphogenetic proteins and noggin: inhibiting and inducing fungiform taste papilla development. Dev. Biol. 297, 198-213.

98. Bar, C., Cohen, I., Zhao, D., Pothula, V., Litskevitch, A., Koseki, H., Zheng, D., and Ezhkova, E. (2019). Polycomb Repressive Complex 1 Controls Maintenance of Fungiform Papillae by Repressing Sonic Hedgehog Expression. Cell Rep. 28, 257-266.e5.

99. McCrickerd, K., and Forde, C.G. (2016). Sensory influences on food intake control: moving beyond palatability. Obes. Rev. 17, 18-29. 
100.Swithers, S.E., and Davidson, T.L. (2008). A role for sweet taste: calorie predictive relations in energy regulation by rats. Behav. Neurosci. 122, 161-173.

101. Hirosawa, M., Laboratory of Cellular Biochemistry, Department of Animal Resource Sciences/Veterinary Medical Sciences, The University of Tokyo, Tokyo 113-8657, Japan, Hayakawa, K., Shiota, K., Tanaka, S., Laboratory of Cellular Biochemistry, Department of Animal Resource Sciences/Veterinary Medical Sciences, The University of Tokyo, Tokyo 113-8657, Japan, Waseda Research Institute for Science and Engineering, Waseda University, Tokyo 169-8555, Japan, and Laboratory of Cellular Biochemistry, Department of Animal Resource Sciences/Veterinary Medical Sciences, The University of Tokyo, Tokyo 113-8657, Japan (2018). Histone O-GlcNAcylation and potential biological functions. OBM genet. 2, 1-1.

102.Konzman, D., Abramowitz, L.K., Steenackers, A., Mukherjee, M.M., Na, H.-J., and Hanover, J.A. (2020). O-GIcNAc: Regulator of Signaling and Epigenetics Linked to X-linked Intellectual Disability. Front. Genet. $11,605263$.

103.Forma, E., Jóźwiak, P., Ciesielski, P., Zaczek, A., Starska, K., Bryś, M., and Krześlak, A. (2018). Impact of OGT deregulation on EZH2 target genes FOXA1 and FOXC1 expression in breast cancer cells. PLoS One 13, e0198351.

104.Lo, P.-W., Shie, J.-J., Chen, C.-H., Wu, C.-Y., Hsu, T.-L., and Wong, C.-H. (2018). O-GlcNAcylation regulates the stability and enzymatic activity of the histone methyltransferase EZH2. Proc. Natl. Acad. Sci. U. S. A. $115,7302-7307$.

105.Sui, B., Chen, D., Liu, W., Wu, Q., Tian, B., Li, Y., Hou, J., Liu, S., Xie, J., Jiang, H., et al. (2020). A novel antiviral IncRNA, EDAL, shields a T309 O-GIcNAcylation site to promote EZH2 lysosomal degradation. Genome Biol. 21, 228.

106.Jiang, M., Xu, B., Li, X., Shang, Y., Chu, Y., Wang, W., Chen, D., Wu, N., Hu, S., Zhang, S., et al. (2019). O-GIcNAcylation promotes colorectal cancer metastasis via the miR-101-O-GlcNAc/EZH2 regulatory feedback circuit. Oncogene 38, 301-316.

107.You, Z., Peng, D., Cao, Y., Zhu, Y., Yin, J., Zhang, G., and Peng, X. (2021). P53 suppresses the progression of hepatocellular carcinoma via miR-15a by decreasing OGT expression and EZH2 stabilization. J. Cell. Mol. Med. 25, 9168-9182.

108.Brien, G.L., Gambero, G., O’Connell, D.J., Jerman, E., Turner, S.A., Egan, C.M., Dunne, E.J., Jurgens, M.C., Wynne, K., Piao, L., et al. (2012). Polycomb PHF19 binds H3K36me3 and recruits PRC2 and demethylase NO66 to embryonic stem cell genes during differentiation. Nat. Struct. Mol. Biol. 19, 1273-1281.

109.Cai, L., Rothbart, S.B., Lu, R., Xu, B., Chen, W.-Y., Tripathy, A., Rockowitz, S., Zheng, D., Patel, D.J., Allis, C.D., et al. (2013). An H3K36 methylation-engaging Tudor motif of polycomb-like proteins mediates PRC2 complex targeting. Mol. Cell 49, 571-582.

110. Ballaré, C., Lange, M., Lapinaite, A., Martin, G.M., Morey, L., Pascual, G., Liefke, R., Simon, B., Shi, Y., Gozani, O., et al. (2012). Phf19 links methylated Lys36 of histone H3 to regulation of Polycomb activity. Nat. Struct. Mol. Biol. 19, 1257-1265.

111. Huang, Z., Cai, L., and Tu, B.P. (2015). Dietary control of chromatin. Curr. Opin. Cell Biol. 34, 69-74.

112. Secco, D., Whelan, J., Rouached, H., and Lister, R. (2017). Nutrient stress-induced chromatin changes in plants. Curr. Opin. Plant Biol. 39, 1-7.

113. Campbell, S.L., and Wellen, K.E. (2018). Metabolic Signaling to the Nucleus in Cancer. Mol. Cell 71, 398-408. 
114. Hobert, O. (2011). Regulation of terminal differentiation programs in the nervous system. Annu. Rev. Cell Dev. Biol. 27, 681-696.

115. Marder, E., and Prinz, A.A. (2002). Modeling stability in neuron and network function: the role of activity in homeostasis. Bioessays 24, 1145-1154.

116. Parrish, J.Z., Kim, C.C., Tang, L., Bergquist, S., Wang, T., Derisi, J.L., Jan, L.Y., Jan, Y.N., and Davis, G.W. (2014). Krüppel mediates the selective rebalancing of ion channel expression. Neuron 82, 537-544.

117. Ryall, J.G., Cliff, T., Dalton, S., and Sartorelli, V. (2015). Metabolic Reprogramming of Stem Cell Epigenetics. Cell Stem Cell 17, 651-662.

118. Miyazawa, H., and Aulehla, A. (2018). Revisiting the role of metabolism during development. Development 145.

119. Lyko, F., Foret, S., Kucharski, R., Wolf, S., Falckenhayn, C., and Maleszka, R. (2010). The honey bee epigenomes: differential methylation of brain DNA in queens and workers. PLoS Biol. 8, e1000506.

120.Kucharski, R., Maleszka, J., Foret, S., and Maleszka, R. (2008). Nutritional control of reproductive status in honeybees via DNA methylation. Science 319, 1827-1830.

121.Ong, Q., Han, W., and Yang, X. (2018). O-GlcNAc as an Integrator of Signaling Pathways. Front. Endocrinol. 9, 599.

122.Ardiel, E.L., McDiarmid, T.A., Timbers, T.A., Lee, K.C.Y., Safaei, J., Pelech, S.L., and Rankin, C.H. (2018). CaMK (CMK-1) and O-GIcNAc transferase (OGT-1) modulate mechanosensory responding and habituation in an interstimulus interval-dependent manner in Caenorhabditis elegans. bioRxiv, 115972.

123.Lagerlöf, O., Slocomb, J.E., Hong, I., Aponte, Y., Blackshaw, S., Hart, G.W., and Huganir, R.L. (2016). The nutrient sensor OGT in PVN neurons regulates feeding. Science 351, 1293-1296.

124.Su, C., and Schwarz, T.L. (2017). O-GIcNAc Transferase Is Essential for Sensory Neuron Survival and Maintenance. J. Neurosci. 37, 2125-2136.

125.Giles, A.C., Desbois, M., Opperman, K.J., Tavora, R., Maroni, M.J., and Grill, B. (2019). A complex containing the O-GICNAc transferase OGT-1 and the ubiquitin ligase EEL-1 regulates GABA neuron function. J. Biol. Chem. 294, 6843-6856.

126.Lagerlöf, O., Hart, G.W., and Huganir, R.L. (2017). O-GlcNAc transferase regulates excitatory synapse maturity. Proc. Natl. Acad. Sci. U. S. A. 114, 1684-1689.

127.Ruan, H.-B., Dietrich, M.O., Liu, Z.-W., Zimmer, M.R., Li, M.-D., Singh, J.P., Zhang, K., Yin, R., Wu, J., Horvath, T.L., et al. (2014). O-GlcNAc transferase enables AgRP neurons to suppress browning of white fat. Cell 159, 306-317.

128. Hwang, H., and Rhim, H. (2019). Acutely elevated O-GlcNAcylation suppresses hippocampal activity by modulating both intrinsic and synaptic excitability factors. Sci. Rep. 9, 7287.

129. Floridi, L. (2005). Is semantic information meaningful data? Philos. Phenomenol. Res. 70, 351-370.

130.Gluckman, P.D., Hanson, M.A., Bateson, P., Beedle, A.S., Law, C.M., Bhutta, Z.A., Anokhin, K.V., Bougnères, P., Chandak, G.R., Dasgupta, P., et al. (2009). Towards a new developmental synthesis: adaptive developmental plasticity and human disease. Lancet 373, 1654-1657.

131.Francis, D., Diorio, J., Liu, D., and Meaney, M.J. (1999). Nongenomic transmission across generations of maternal behavior and stress responses in the rat. Science 286, 1155-1158. 
132. Champagne, F.A. (2013). Epigenetics and developmental plasticity across species. Dev. Psychobiol. 55, 33-41.

133. Shannon, C.E. (1948). A mathematical theory of communication. The Bell System Technical Journal 27 , 379-423.

134. Reinagel, P. (2000). Information theory in the brain. Curr. Biol. 10, R542-4.

135.Smith, J.M. (2000). The Concept of Information in Biology. Philos. Sci. 67, 177-194.

136. Fabris, F. (2009). Shannon information theory and molecular biology. J. Interdiscip. Math. 12, 41-87.

137.Musselman, L.P., and Kühnlein, R.P. (2018). Drosophila as a model to study obesity and metabolic disease. J. Exp. Biol. 221.

138. Shiraiwa, T., and Carlson, J.R. (2007). Proboscis extension response (PER) assay in Drosophila. J. Vis. Exp., 193.

139.Andrews, S., and Others (2010). FastQC: a quality control tool for high throughput sequence data.

140.Dobin, A., Davis, C.A., Schlesinger, F., Drenkow, J., Zaleski, C., Jha, S., Batut, P., Chaisson, M., and Gingeras, T.R. (2013). STAR: ultrafast universal RNA-seq aligner. Bioinformatics 29, 15-21.

141.Anders, S., Pyl, P.T., and Huber, W. (2015). HTSeq--a Python framework to work with high-throughput sequencing data. Bioinformatics 31, 166-169.

142.Love, M.I., Huber, W., and Anders, S. (2014). Moderated estimation of fold change and dispersion for RNA-seq data with DESeq2. Genome Biol. 15, 550.

143.Shen, L., and Sinai, M. (2013). GeneOverlap: Test and visualize gene overlaps. R package version 1, 2013.

144.Quinlan, A.R., and Hall, I.M. (2010). BEDTools: a flexible suite of utilities for comparing genomic features. Bioinformatics 26, 841-842.

145.Wickham, H. (2016). ggplot2: Elegant Graphics for Data Analysis (Springer International Publishing).

146. Kolde, R. (2012). Pheatmap: pretty heatmaps. R package version.

147.Wilkinson, L. (2012). Exact and approximate area-proportional circular Venn and Euler diagrams. IEEE Trans. Vis. Comput. Graph. 18, 321-331.

148. Blighe, K. (2019). EnhancedVolcano: Publication-ready Volcano Plots With Enhanced Colouring and Labeling. R Package Version 120. 2019.

149.Marshall, O.J., and Brand, A.H. (2015). damidseq_pipeline: an automated pipeline for processing DamID sequencing datasets. Bioinformatics 31, 3371-3373.

150.Langmead, B., and Salzberg, S.L. (2012). Fast gapped-read alignment with Bowtie 2. Nat. Methods 9 , 357-359.

151.Ramírez, F., Ryan, D.P., Grüning, B., Bhardwaj, V., Kilpert, F., Richter, A.S., Heyne, S., Dündar, F., and Manke, T. (2016). deepTools2: a next generation web server for deep-sequencing data analysis. Nucleic Acids Res. 44, W160-5.

152.Heinz, S., Benner, C., Spann, N., Bertolino, E., Lin, Y.C., Laslo, P., Cheng, J.X., Murre, C., Singh, H., and 
Glass, C.K. (2010). Simple combinations of lineage-determining transcription factors prime cis-regulatory elements required for macrophage and B cell identities. Mol. Cell 38, 576-589.

153.Benjamini, Y., and Hochberg, Y. (1995). Controlling the False Discovery Rate: A Practical and Powerful Approach to Multiple Testing. J. R. Stat. Soc. Series B Stat. Methodol. 57, 289-300.

154.Raudvere, U., Kolberg, L., Kuzmin, I., Arak, T., Adler, P., Peterson, H., and Vilo, J. (2019). g:Profiler: a web server for functional enrichment analysis and conversions of gene lists (2019 update). Nucleic Acids Research 47, W191-W198.

155.Chen, E.Y., Tan, C.M., Kou, Y., Duan, Q., Wang, Z., Meirelles, G.V., Clark, N.R., and Ma'ayan, A. (2013). Enrichr: interactive and collaborative HTML5 gene list enrichment analysis tool. BMC Bioinformatics 14, 128.

156.Kuleshov, M.V., Jones, M.R., Rouillard, A.D., Fernandez, N.F., Duan, Q., Wang, Z., Koplev, S., Jenkins, S.L., Jagodnik, K.M., Lachmann, A., et al. (2016). Enrichr: a comprehensive gene set enrichment analysis web server 2016 update. Nucleic Acids Res. 44, W90-7.

157.Xie, Z., Bailey, A., Kuleshov, M.V., Clarke, D.J.B., Evangelista, J.E., Jenkins, S.L., Lachmann, A., Wojciechowicz, M.L., Kropiwnicki, E., Jagodnik, K.M., et al. (2021). Gene Set Knowledge Discovery with Enrichr. Curr Protoc 1, e90. 
bioRxiv preprint doi: https://doi.org/10.1101/2021.12.17.473205; this version posted December 19, 2021. The copyright holder for this preprint Vaziri'(which was not certified by peer review) is the author/funder, who has granted bioRxiv a license to display the preprint in perpetuity. It is made

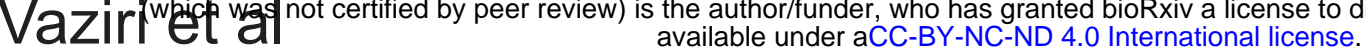

\section{Figure 1}

A

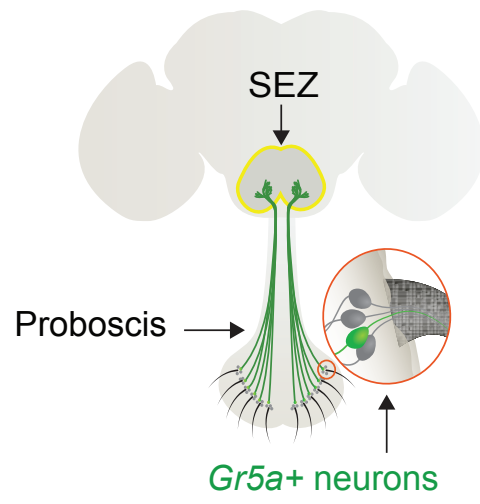

B

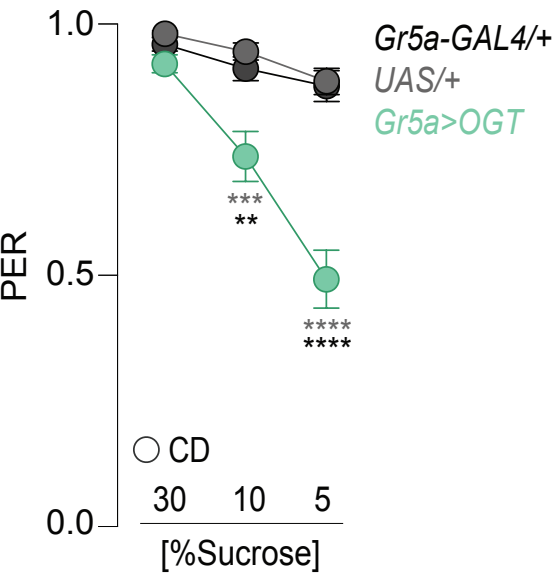

C

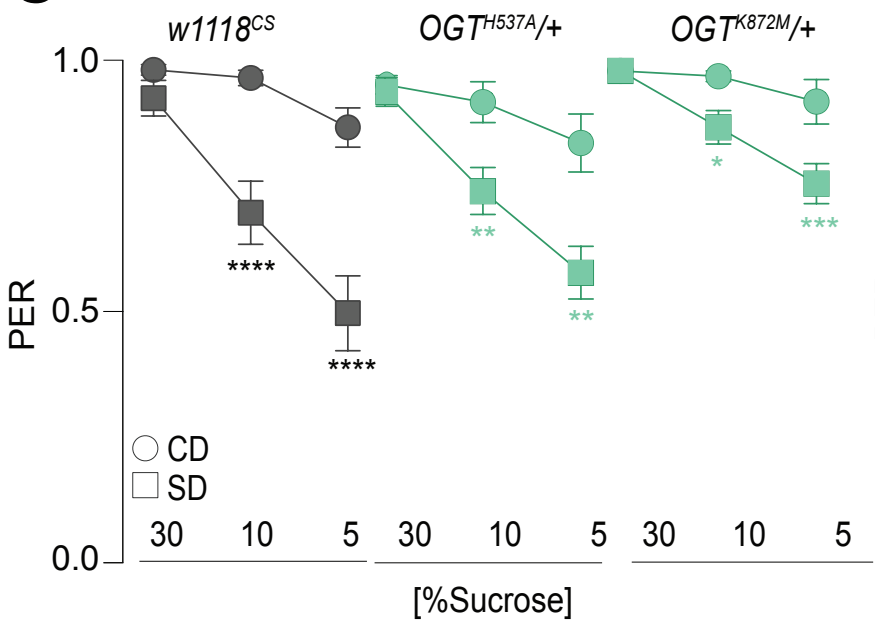

D

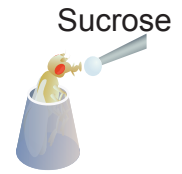

+vehicle +10uM OSMI-1

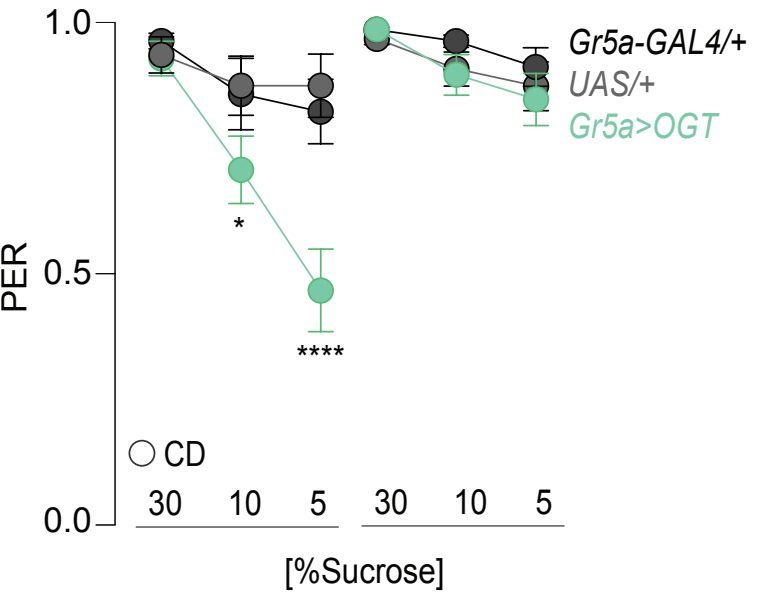




\section{Vaziri et al}

Figure 2

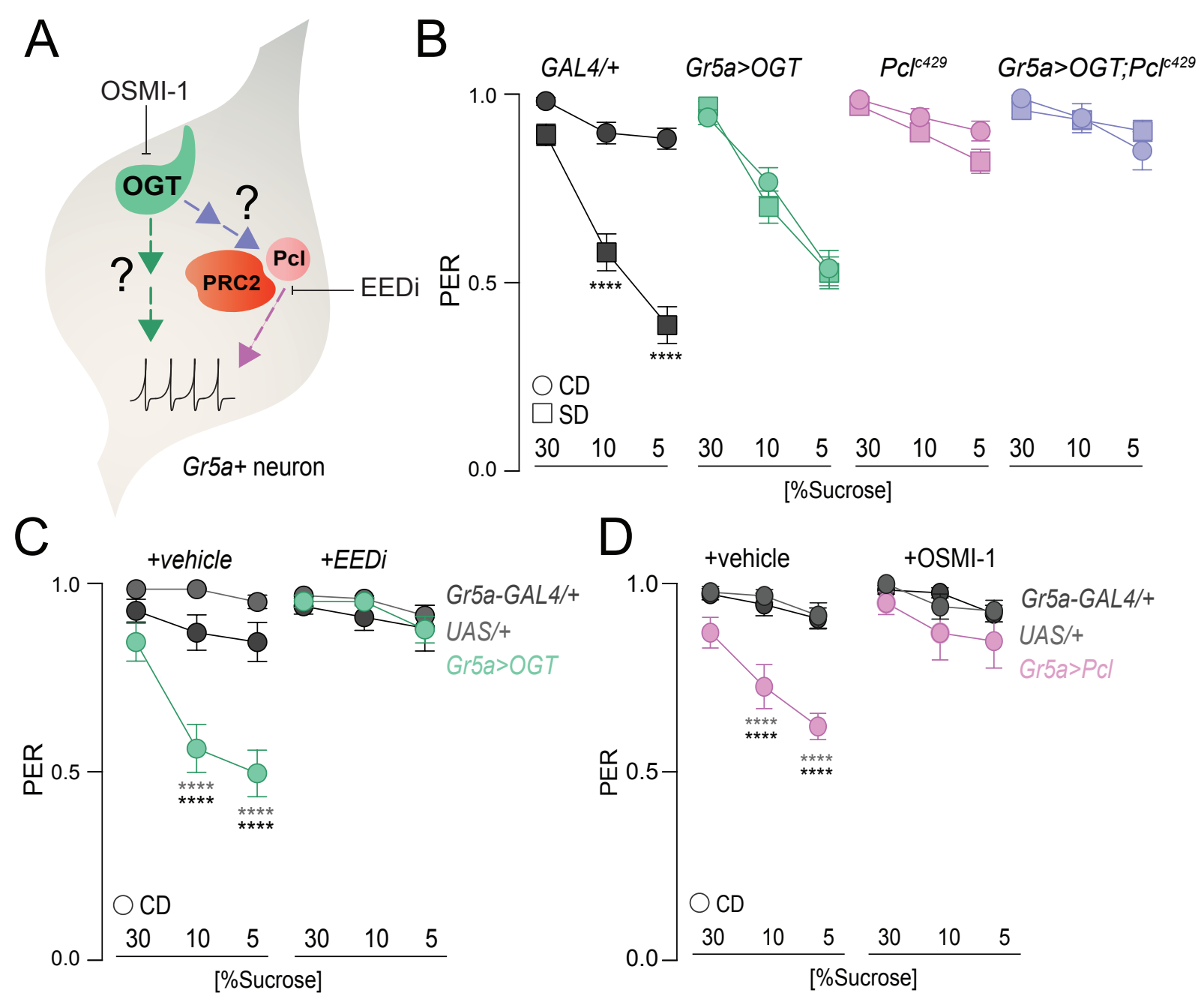




\section{Vaziri et al}

Figure 3
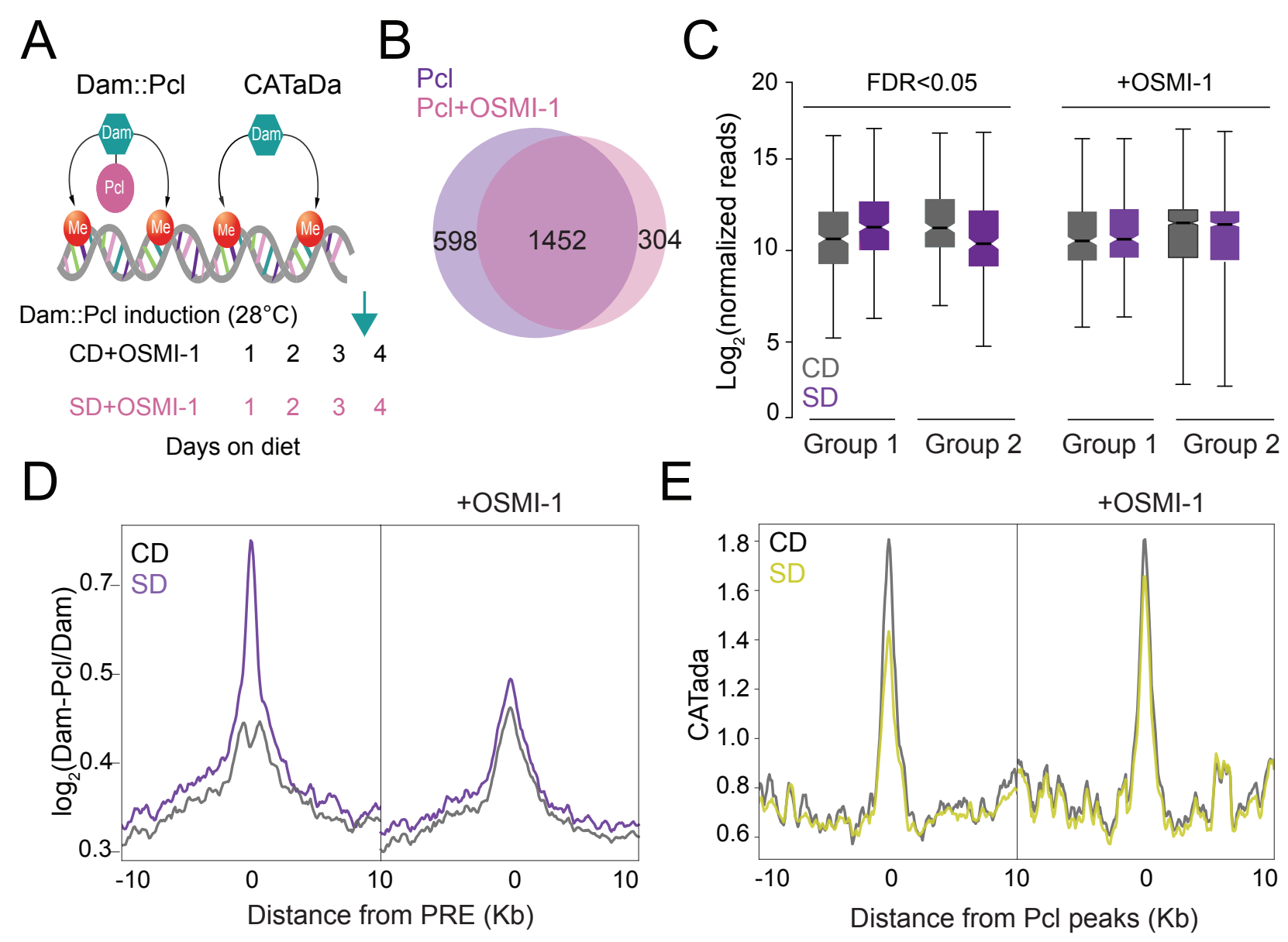
A

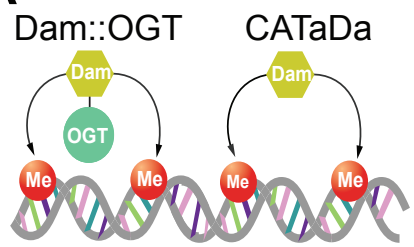

Dam::OGT induction $\left(28^{\circ} \mathrm{C}\right)$

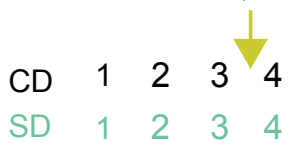

Days on diet

D

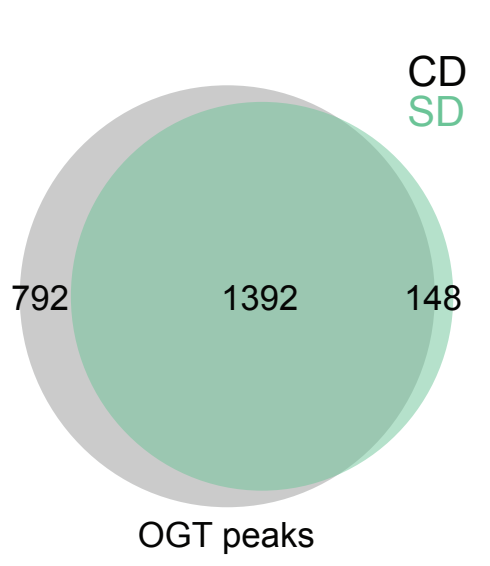

F

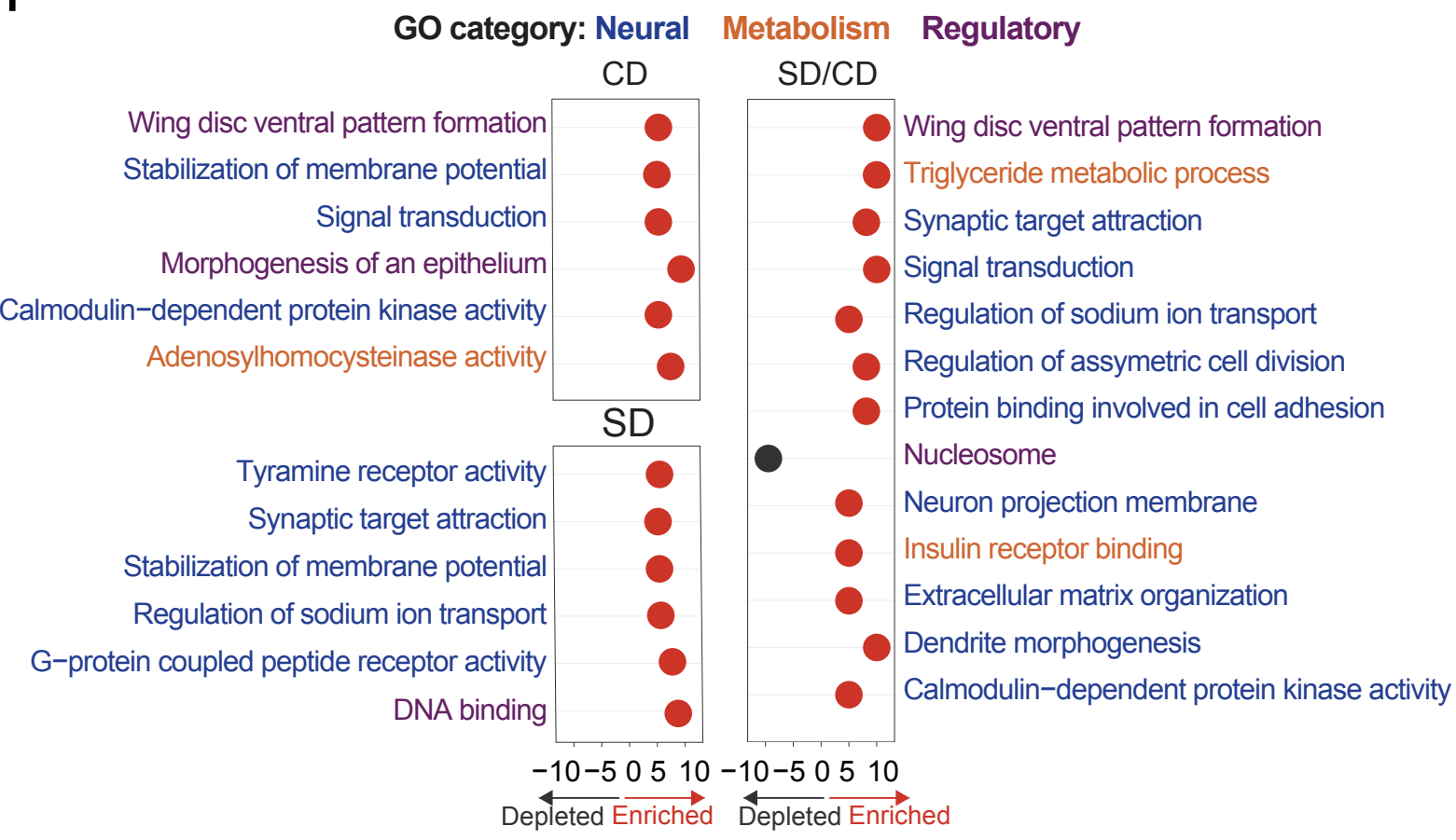

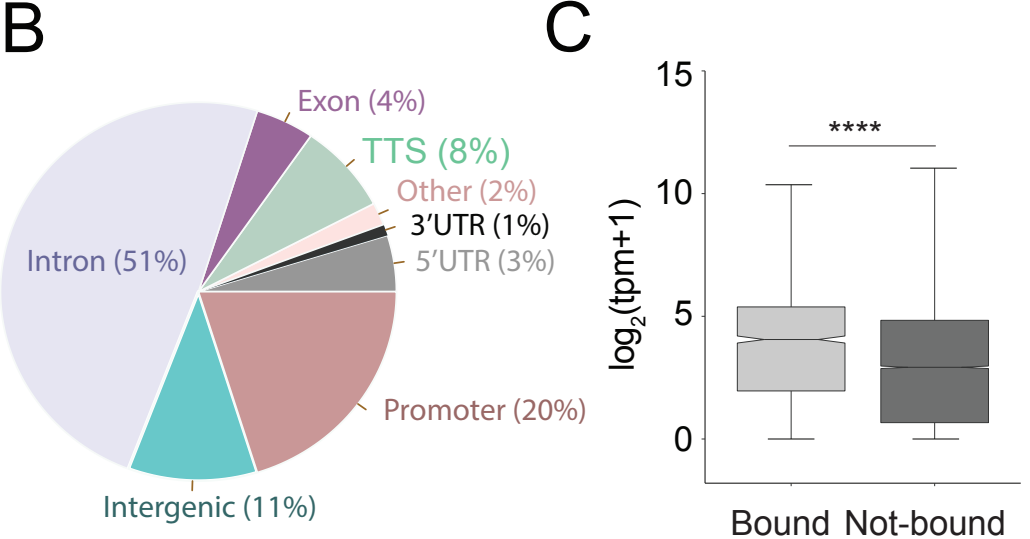

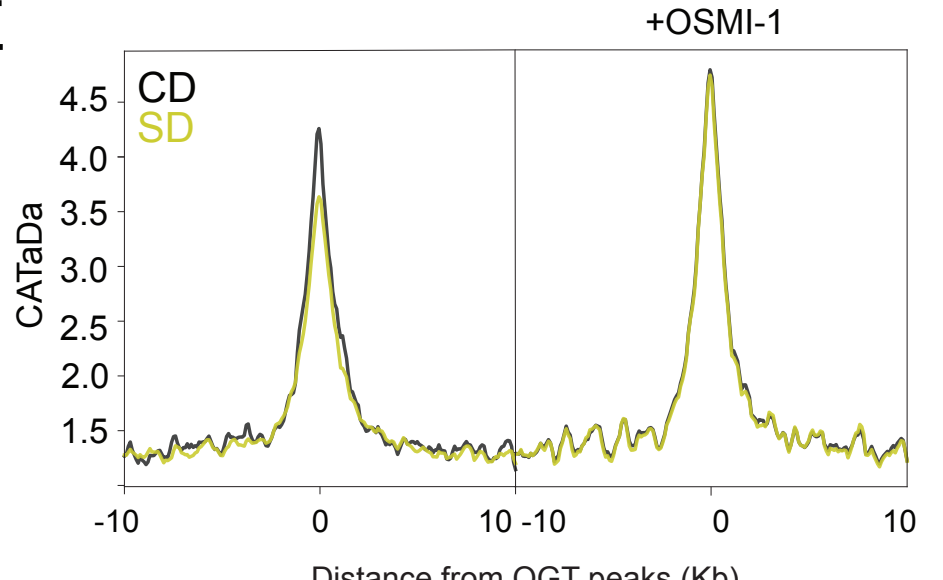




\section{Vaziri et al}

Figure 5
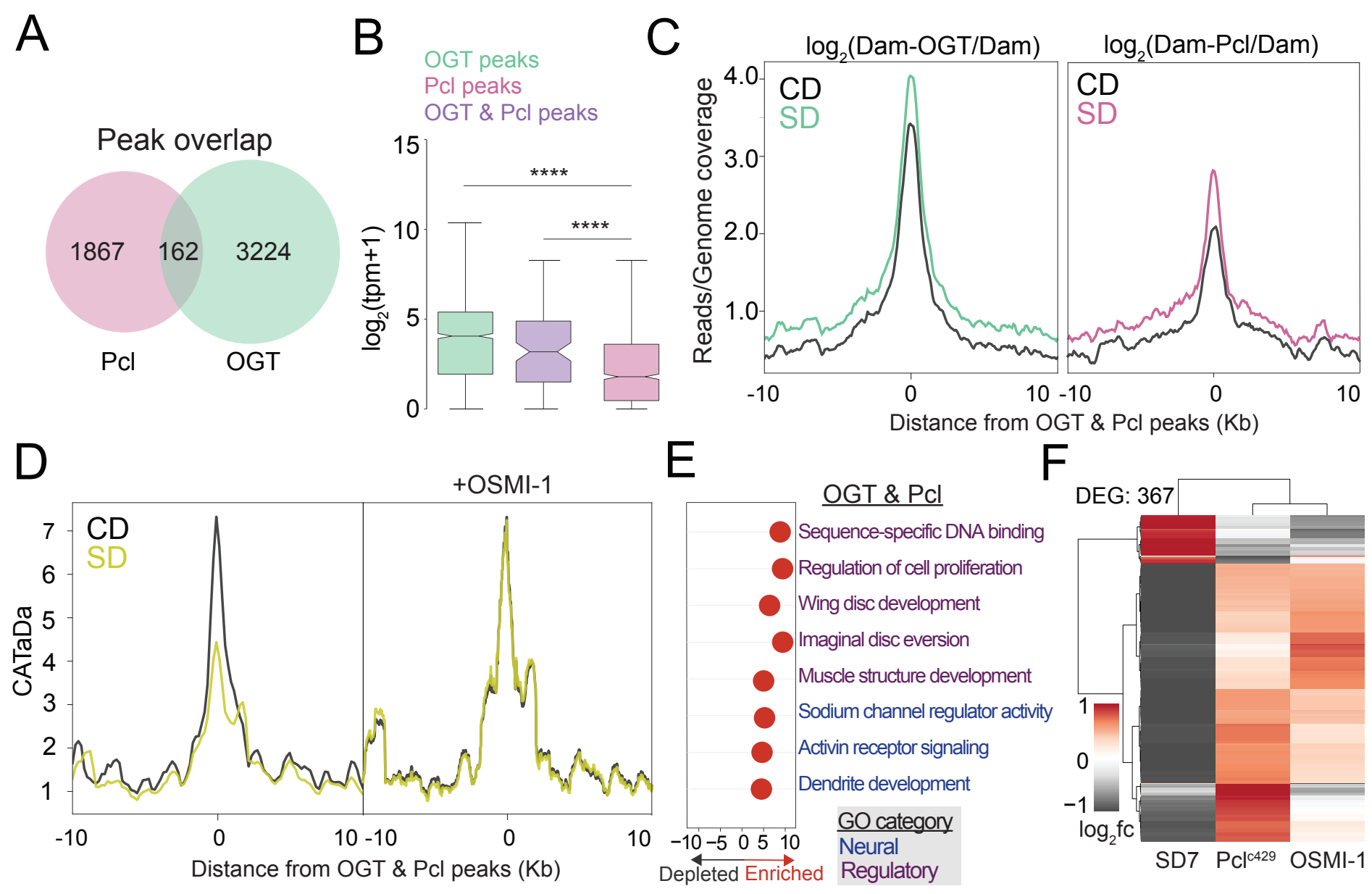


\section{Vaziri et al}

\section{Figure 6}

A

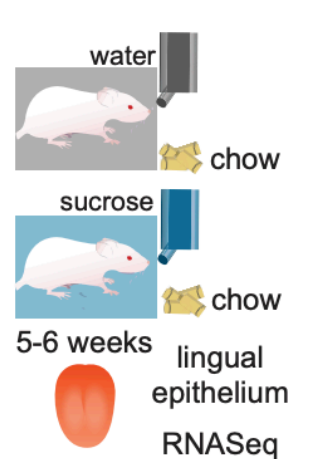

E

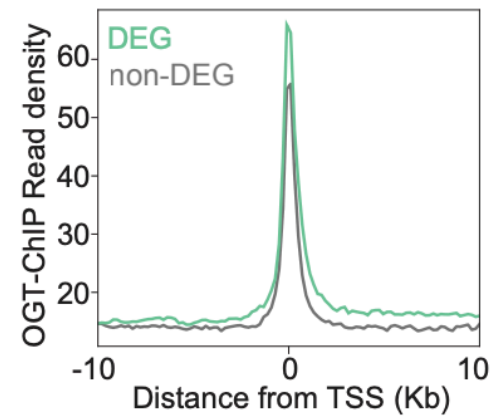

B

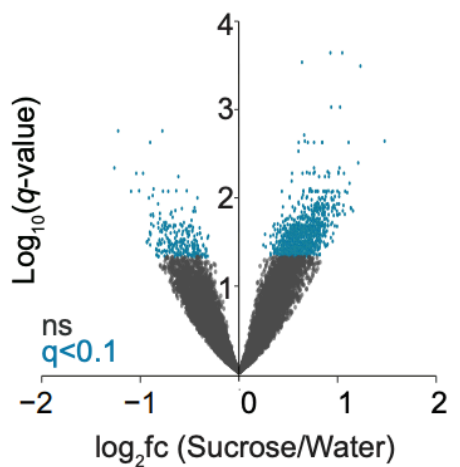

$\mathrm{F}$

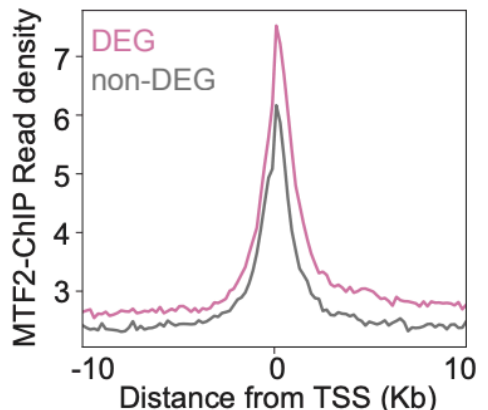

C

Cellular component organization

Metabolic process

Positive regulation of biological process

System development Cell differentiation

$$
\text { Cell death }
$$

Cellular response to chemical stimuli

Regulation of signaling

$$
\begin{gathered}
0 \quad 510152025 \\
-\log _{10}(q \text {-value) }
\end{gathered}
$$

D

Sucrose/Water DEG

OGT peaks

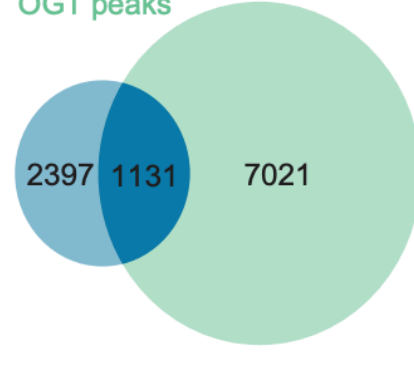

G

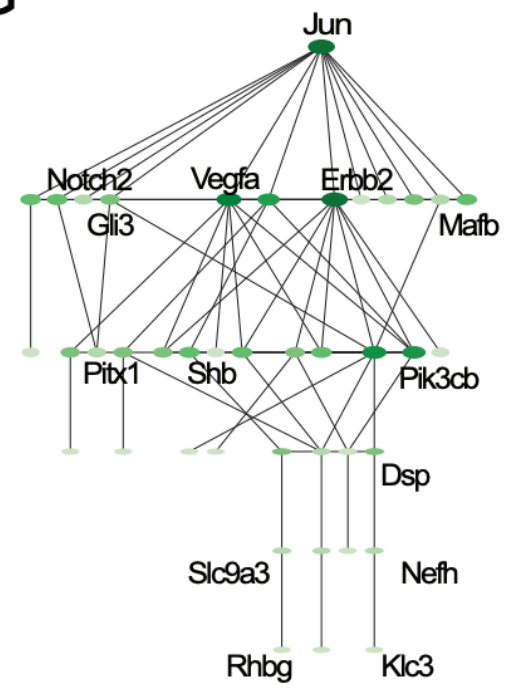

$\mathrm{H}$

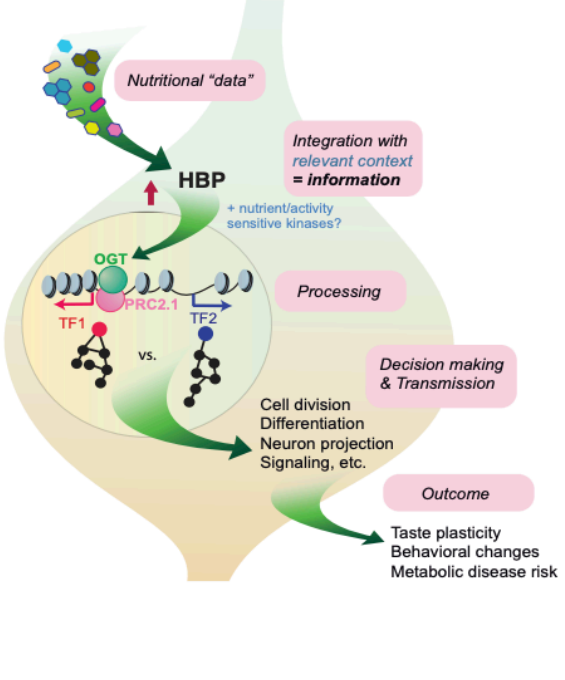


bioRxiv preprint doi: https://doi.org/10.1101/2021.12.17.473205; this version posted December 19, 2021. The copyright holder for this preprint (which was not certified by peer review) is the author/funder, who has granted bioRxiv a license to display the preprint in perpetuity. It is made $V$ aziri (which was not certified by peer review) is the author/funder, who has granted bioRxiv a license to dis
available under aCC-BY-NC-ND 4.0 International license.

\section{Supplement 1}

A $B$
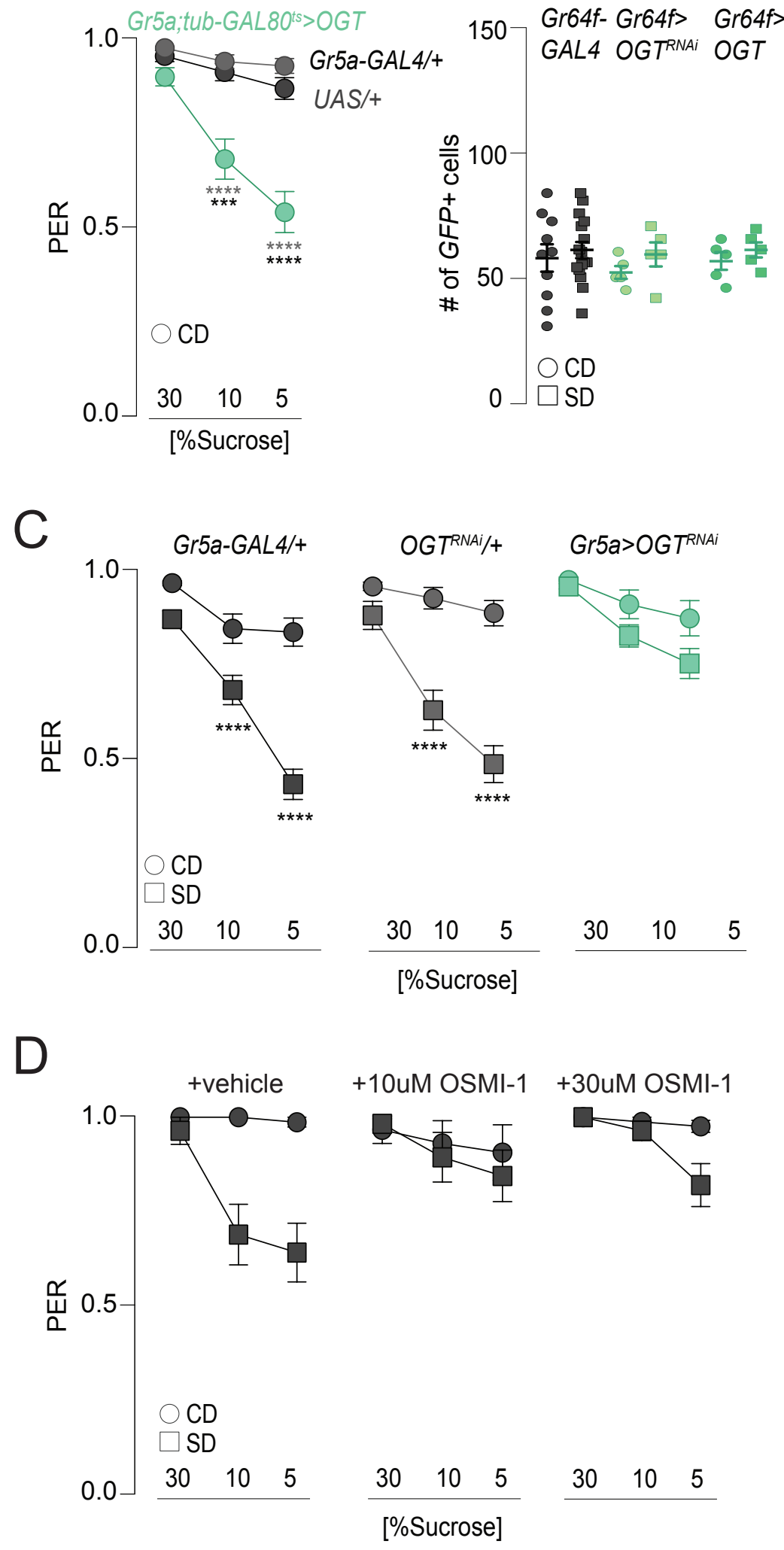
bioRxiv preprint doi: https://doi.org/10.1101/2021.12.17.473205; this version posted December 19, 2021. The copyright holder for this preprint V7i (Mhi prasigdt certified by peer review) is the author/funder, who has granted bioRxiv a license to display the preprint in perpetuity. It is made Supplement 2 available under aCC-BY-NC-ND 4.0 International license.

A

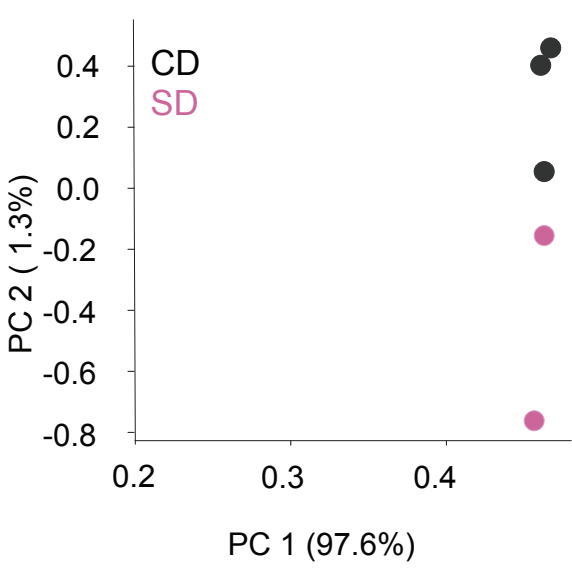

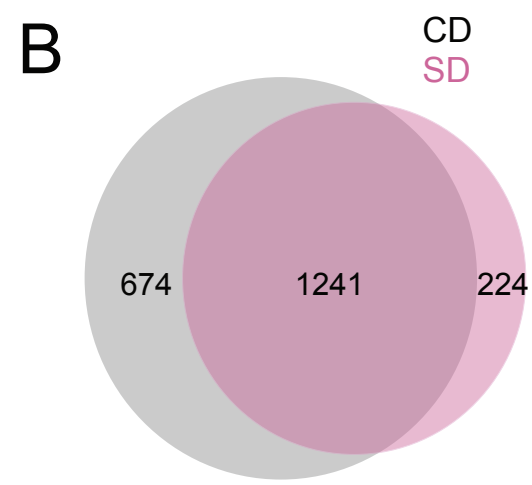

Dam-Pcl +10uM OSMI-1

C

Background Shared Pcl and Pcl+OSMI-1

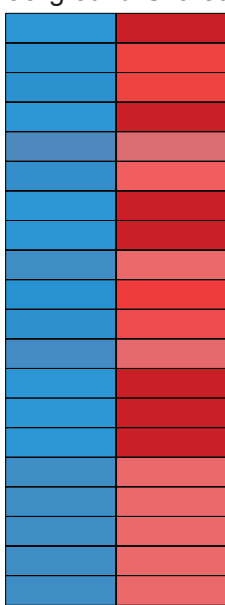

Under-representation -5

sequence-specific DNA binding, GO:0043565

gliogenesis, GO:0042063

cell development, GO:0048468

anatomical structure development, GO:0048856

mitochondrial respiratory chain, GO:0005746

synaptic target attraction, GO:0016200

sensory perception of sound, GO:0007605

neuroendocrine cell differentiation, GO:0061101

negative regulation of photoreceptor cell differen

wing disc development, GO:0035220

developmental process, GO:0032502

intracellular cholesterol transport, GO:0032367

leg disc proximal/distal pattern formation, GO:000

cell fate specification, GO:0001708

blastoderm segmentation, GO:0007350

anterior region determination, GO:0007355

male courtship behavior, orientation prior to leg

elongation of arista core, GO:0035015

sex-specific pigmentation, GO:0048071

enterocyte differentiation, GO:1903703

$D$

\section{Background $\mathrm{Pcl} \quad \mathrm{Pcl}+\mathrm{OSMl}-1$}

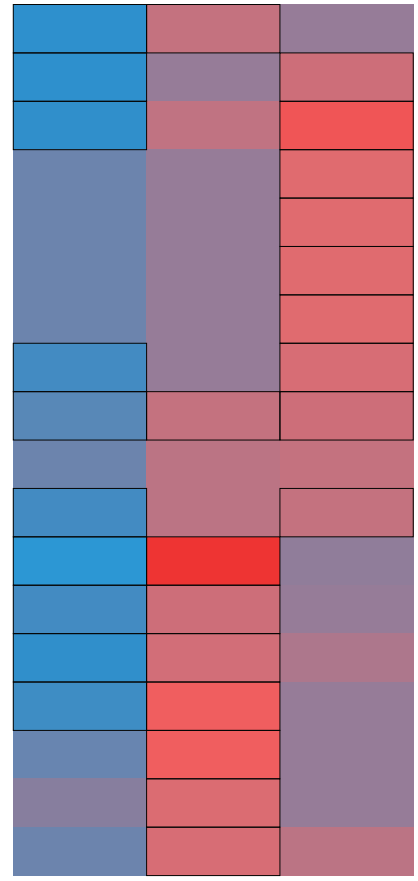

gliogenesis, GO:0042063

AT DNA binding, GO:0003680

homophilic cell adhesion via plasma membrane adhesion molecules, GO:0007156

histidyl-tRNA aminoacylation, GO:0006427

polyamine biosynthetic process, GO:0006596

catecholamine catabolic process, GO:0042424

D-gluconate catabolic process, GO:0046177

negative regulation of stress-activated protein kinase signaling cascade, GO:0070303

dopamine receptor signaling pathway, GO:0007212

mRNA splice site selection, GO:0006376

sarcolemma, GO:0042383

sensory perception of sound, GO:0007605

male courtship behavior, orientation prior to leg tapping and wing vibration, GO:0016543

wing disc development, GO:0035220

antimicrobial humoral response, GO:0019730

GGA codon-amino acid adaptor activity, GO:0033463

detection of chemical stimulus, GO:0009593

DNA damage checkpoint, GO:0000077

Under-representation -5

5 Over-representation 


\section{Vaziri et al}

Supplement 3

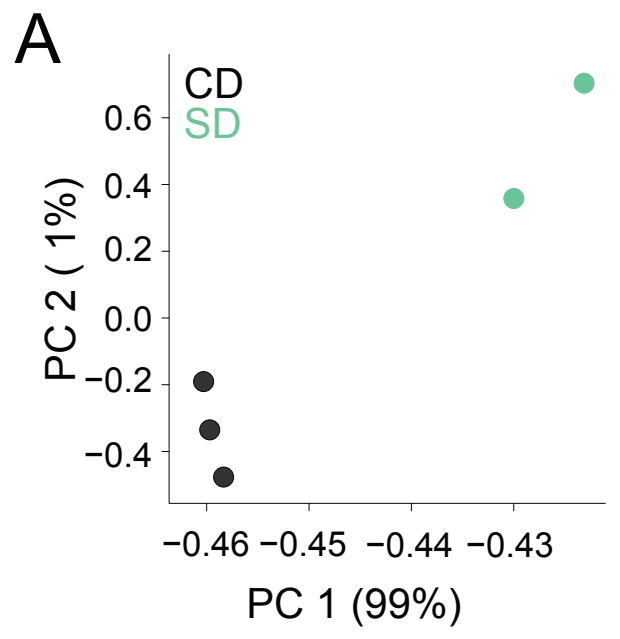

B

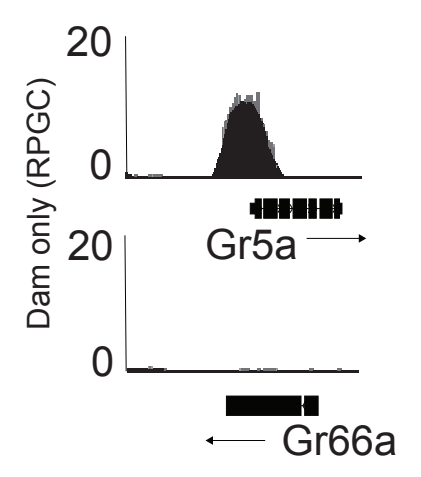

C

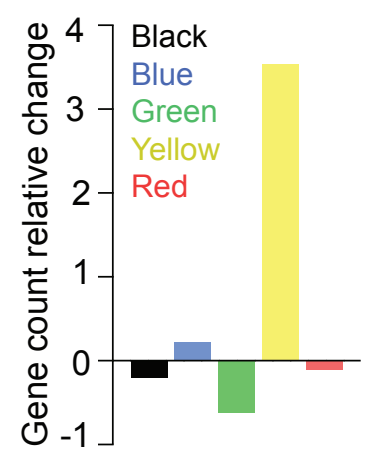


bioRxiv preprint doi: https://doi.org/10.1101/2021.12.17.473205; this version posted December 19, 2021. The copyright holder for this preprint $\checkmark$ Jirich wag not certified by peer review) is the author/funder, who has granted bioRxiv a license to display the preprint in perpetuity. It is made aZIrl et al available under aCC-BY-NC-ND 4.0 International license.

\section{Supplement 4}

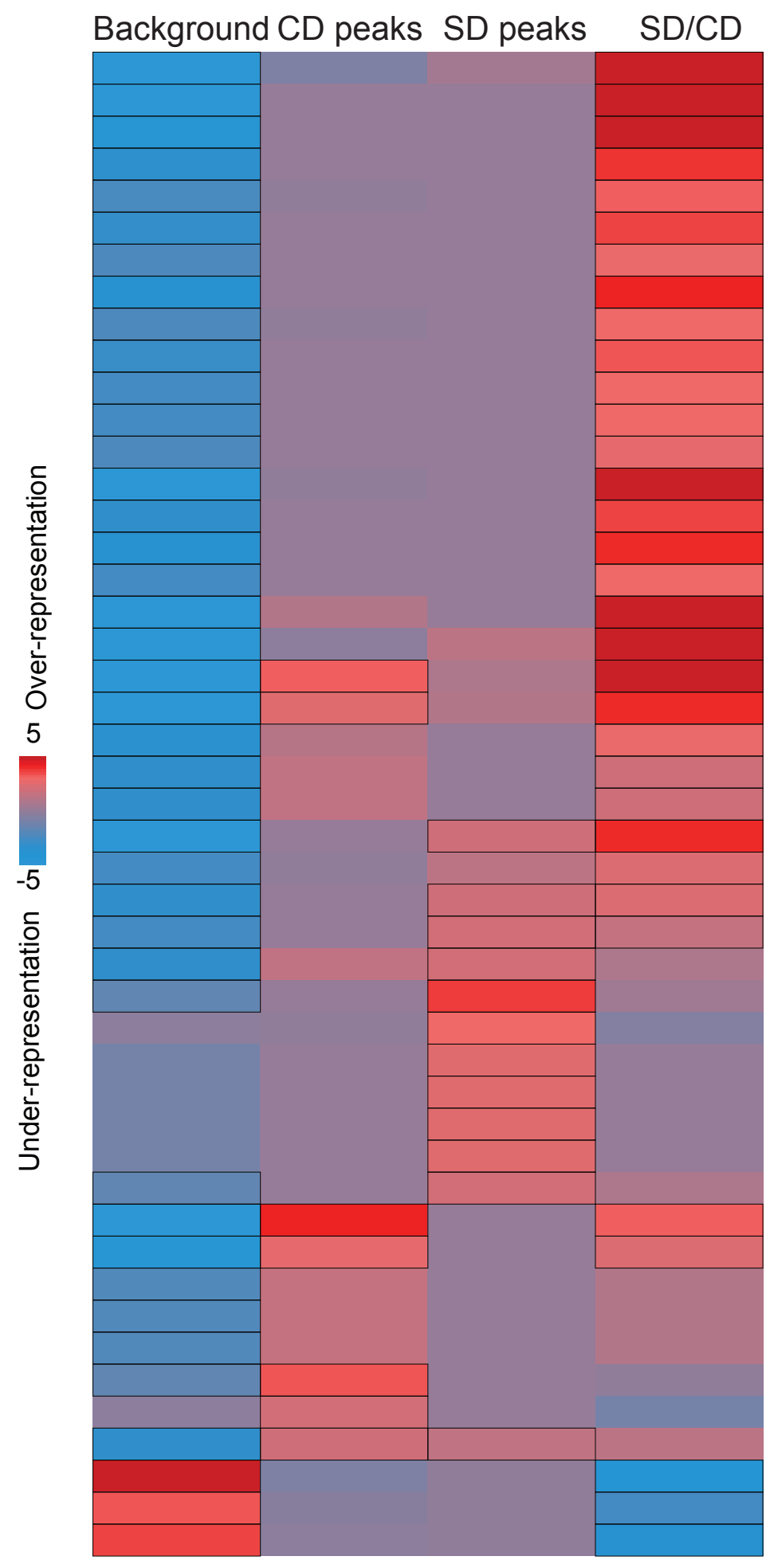

\section{OGT targets GO Terms}

dendrite morphogenesis, GO:0048813

peptidyl-tyrosine phosphorylation, GO:0018108

negative regulation of $\mathrm{R} 7$ cell differentiation, GO:0045677

larval visceral muscle development, GO:0007523

extracellular matrix organization, GO:0030198

protein binding involved in cell-cell adhesion, GO:0098632

insulin receptor binding, GO:0005158

cellular zinc ion homeostasis, GO:0006882

positive regulation of antibacterial peptide biosynthetic process, GO:0006963

regulation of heart contraction, GO:0008016

nucleotide catabolic process, GO:0009166

vascular endothelial growth factor receptor binding, GO:0005172

diacylglycerol kinase activity, GO:0004143

intracellular, GO:0005622

regulation of asymmetric cell division, GO:0009786

renal system process, GO:0003014

BMP binding, GO:0036122

triglyceride metabolic process, GO:0006641

signal transduction, GO:0007165

wing disc dorsal/ventral pattern formation, GO:0048190

cytoskeleton, GO:0005856

response to endoplasmic reticulum stress, GO:0034976

organic acid metabolic process, GO:0006082

cardioblast cell fate specification, GO:0042685

synaptic target attraction, GO:0016200

neuron projection membrane, GO:0032589

regulation of sodium ion transport, GO:0002028

myosin VI complex, GO:0031476

tyramine receptor activity, GO:0008226

DNA binding, bending, GO:0008301

G-protein coupled peptide receptor activity, GO:0008528

peptidyl-pyrromethane cofactor linkage, GO:0018160

phosphopyruvate hydratase activity, GO:0004634

ribonuclease inhibitor activity, GO:0008428

DNA N-glycosylase activity, GO:0019104

Cul5-RING ubiquitin ligase complex, GO:0031466

morphogenesis of an epithelium, GO:0002009

calmodulin-dependent protein kinase activity, GO:0004683

regulation of myofibril size, GO:0014881

NMDA glutamate receptor activity, GO:0004972

PAN complex, GO:0031251

adenosylhomocysteinase activity, GO:0004013

structural constituent of chitin-based cuticle, GO:0005214

stabilization of membrane potential, GO:0030322

cytosolic ribosome, GO:0022626

mitochondrial translation, GO:0032543

nucleosome, GO:0000786 
Vaziri et al

Supplement 5

A
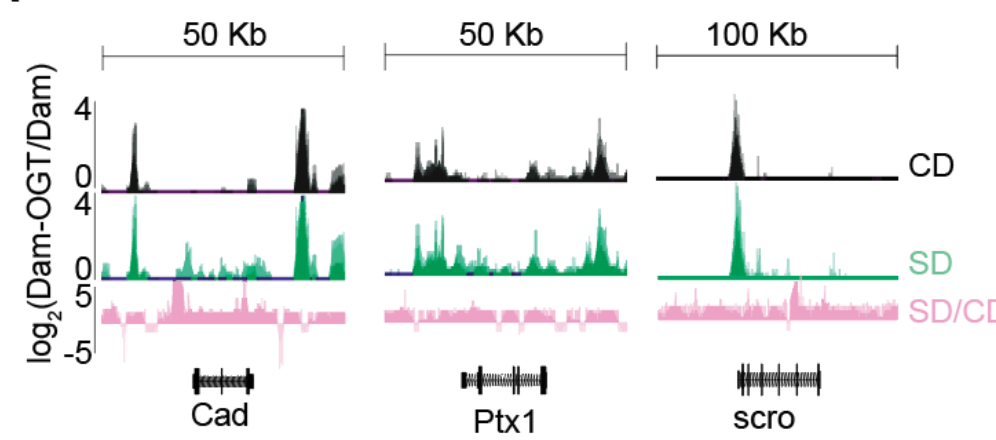

B

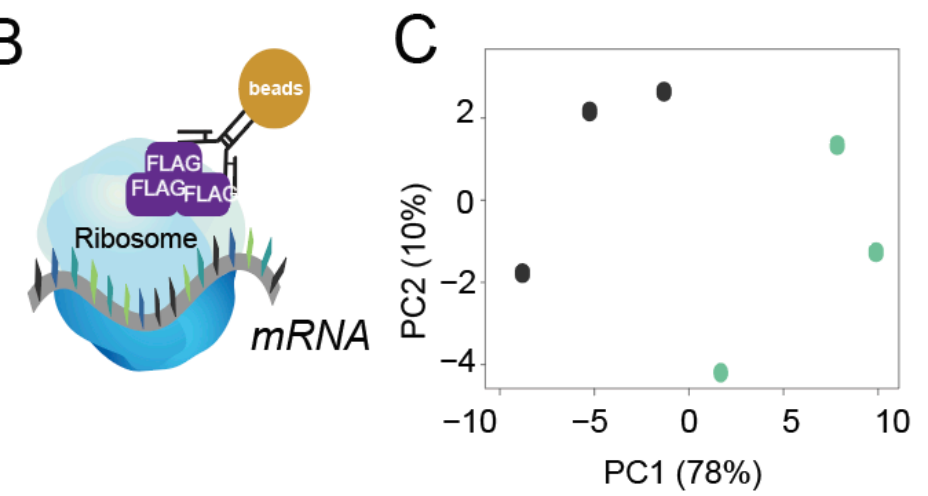

D
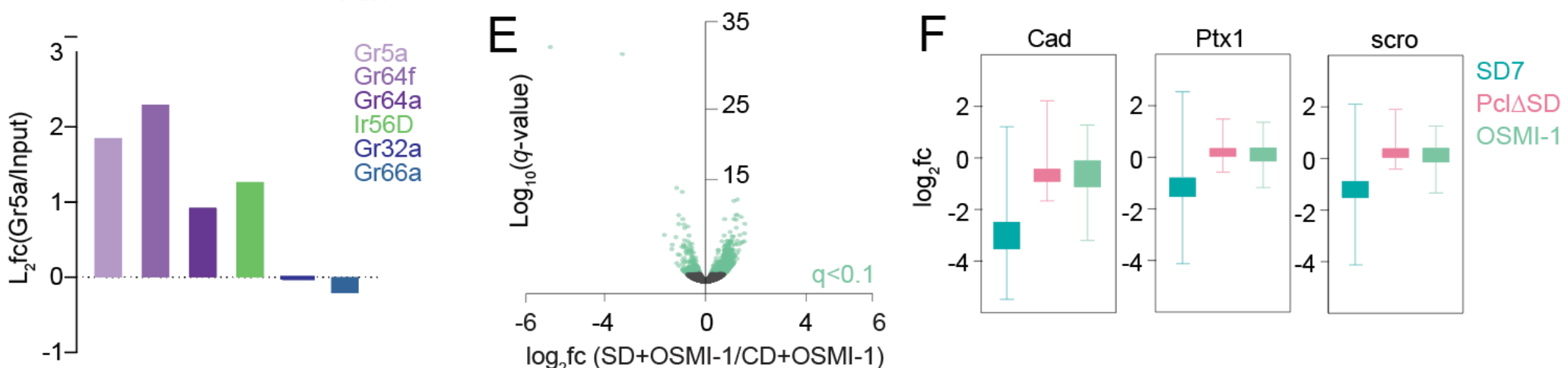
$\log _{2} \mathrm{fc}(\mathrm{SD}+\mathrm{OSMI}-1 / \mathrm{CD}+\mathrm{OSMI}-1)$ 

available under aCC-BY-NC-ND 4.0 International license.

\section{Supplement 6}

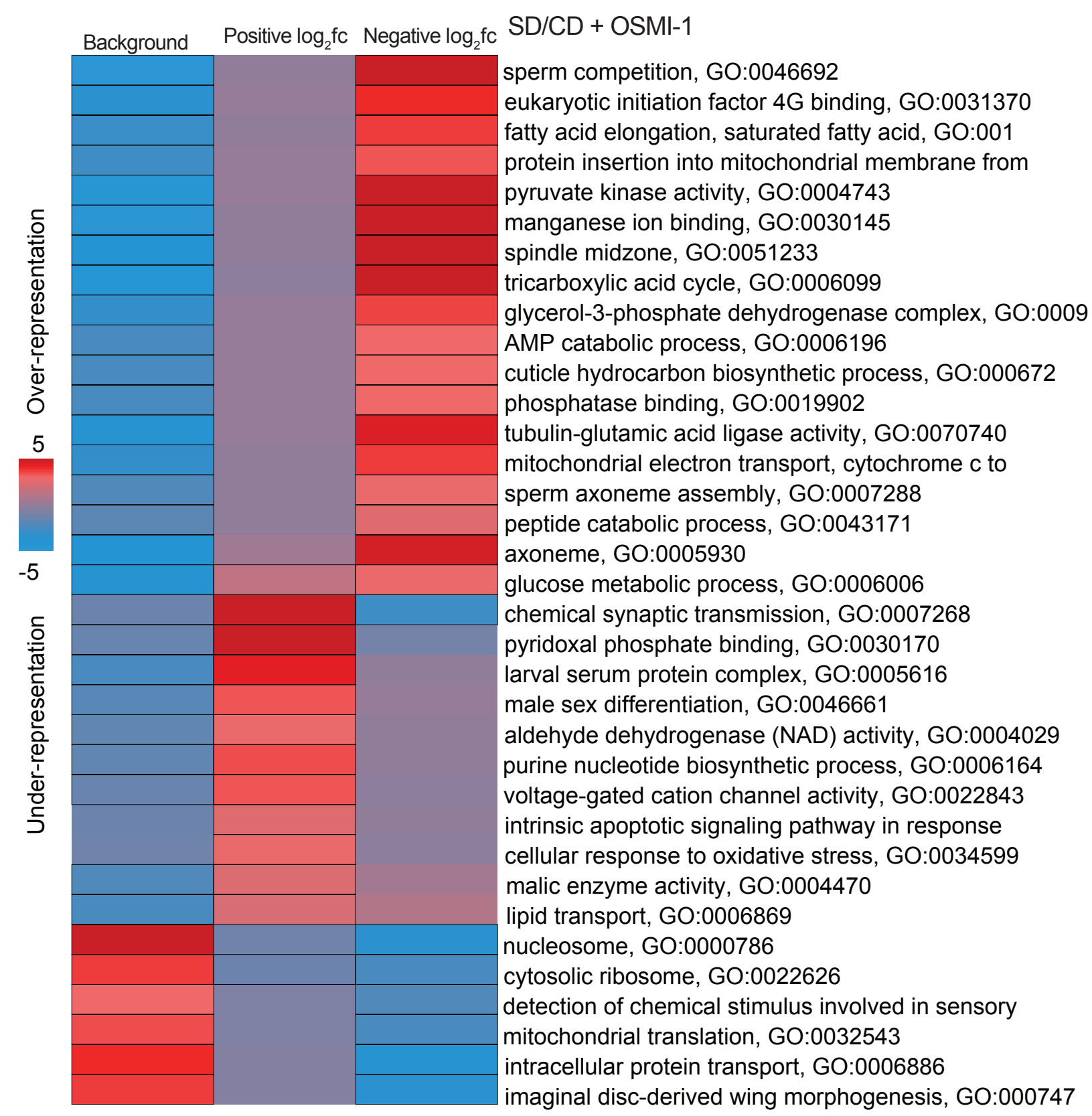




\section{Figure Legends}

\section{Figure 1: OGT Activity Directs Sensory Responses to Sweetness}

(A) Schematic of sweet taste neurons in Drosophila melanogaster (left) and of Proboscis Extension Response (PER; right). (B-D) Proboscis extension response (PER) to stimulation of the labellum with 30, 10, and 5\% sucrose ( $x$ axis) of age-matched male (B) Gr5a>OGT (green) and transgenic controls (shades of gray) on a control diet, $n=36-48$, Kruskal-Wallis Dunn's multiple comparisons, comparisons to transgenic controls. (C) $w 1118^{c s}, O G T^{H 537 A} /+, O G T^{K 872 M} /+$ flies on a control (CD) or sugar diet (SD), $\mathrm{n}=29-33$, Kruskal-Wallis Dunn's multiple comparisons, comparisons to the control diet of each genotype. (D) Gr5a>OGT (green) and transgenic controls (shades of gray) on a sugar diet supplemented with OSMI or vehicle, $n=19-22$, Kruskal-Wallis test with Dunn's multiple comparisons, comparisons to transgenic controls. In all panels, flies were on a CD (circle) or SD (square) diet for 7 days. Data are shown as means \pm SEM. ${ }^{* * * *} p<0.0001,{ }^{* * *} p<0.001,{ }^{* *} p<0.01$, and ${ }^{*} p$ $<0.05$.

\section{Figure 2: OGT and PRC2.1 act in concert to tune sweet taste in response to diet}

(A) Schematic of hypotheses tested in this figure. (B-D) Proboscis extension response (PER) to stimulation of the labellum with 30,10 , and $5 \%$ sucrose ( $x$ axis) of age-matched male $(B)$ Gr5a>OGT;Pc/ ${ }^{429}$ (purple), Gr5a>OGT (green), $P C l^{1429} /+$ (pink), and transgenic controls (gray) on CD or SD, $\mathrm{n}=20-37$, Kruskal-Wallis test with Dunn's multiple comparisons, comparisons to CD of each genotype. (C) Gr5a>OGT (green) and transgenic controls (shades of gray) on a CD supplemented with vehicle (10\% DMSO) or 8 uM EEDi , $n=20-22$, Kruskal-Wallis test with Dunn's multiple comparisons, comparisons to transgenic controls. (D) $\mathrm{Gr} 5 \mathrm{a}>\mathrm{Pcl}$ (pink) and transgenic controls (shades of gray) on CD supplemented with vehicle (10\% DMSO) or $10 \mathrm{uM} \mathrm{OSMI-1,}$ $\mathrm{n}=18-23$, Kruskal-Wallis test with Dunn's multiple comparisons, comparisons to transgenic controls. In all panels, flies were on a control (circle) or sugar (square) diet for 7 days. Data are shown as means \pm SEM. ${ }^{* * * *} P<0.0001,{ }^{* * *} P<0.001,{ }^{* *} P<0.01$, and ${ }^{*} P<0.05$.

Figure 3: The nutrient-dependent chromatin redistribution of $P c /$ is contingent on OGT

(A) Design of the Targeted Dam-ID (TaDa) of Dam::Pcl and Dam (CATaDa) experiments. (B) Overlap of $\log 2($ Dam::Pcl/Dam) chromatin occupancy peaks with (pink) and without (purple) OSMl-1 (find_peaks, q<0.01). (C) The median and variance of log2(Dam::Pcl/Dam) reads with (right) and without (left) OSMI-1 for genes bound on CD and SD. Genes are grouped into higher (group 1) or lower (group 2) Pcl binding on SD, FDR $<0.05$ is presented for differential binding and not peak calling. (D) Average log2(Dam::Pcl/Dam) signal on a CD (gray) or SD (purple) supplemented with (right) or without (left) OSMI-1 centered at PREs. (E) Average 
CATaDa signal on a CD (gray) and SD (yellow) supplemented with (right) or without (left) OSMI-1 centered at PCl peaks.

\section{Figure 4: OGT decorates the chromatin of the sweet taste neurons}

(A) Design of Targeted Dam-ID (TaDa) of Dam::OGT and Dam (CATaDa) experiments. Age-matched Gr5a;tubulin-GAL80 ${ }^{\text {ts }}>$ UAS-LT3-Dam::OGT and Gr5a;tubulin-GAL80 ${ }^{\text {ts }}>$ UAS-LT3-Dam flies were placed on a $\mathrm{CD}$ or $\mathrm{SD}$ for 3 days at $20^{\circ}$ to $21^{\circ} \mathrm{C}$ and then switched to $28^{\circ} \mathrm{C}$ between days 3 and 4 to induce Dam expression. (B) Annotation of OGT chromatin occupied regions using HOMER (see Methods). (C) The distribution of RNA reads normalized as transcripts per million (TPMs) of genes occupied by OGT and genes without OGT occupancy on CD (D) Overlap of log2(Dam::OGT/Dam) chromatin binding peaks of CD (gray) and SD (green) (find_peaks, q<0.01). (E) Average CATaDa signal on a CD (gray) and SD (yellow) centered at OGT peaks with (right) and without (left) OSMI-1. (F) iPAGE summary plots for OGT peaks on a control (top left), sugar (bottom left), and the delta of sugar and control diet (right). Text in blue represents neural GO terms, orange represents metabolic GO terms, and purple represents regulatory GO terms.

\section{Figure 5: OGT and PRC2.1 mark nutrient-sensitive chromatin in the sweet taste cells}

(A) Overlap of log2(Dam::Pcl/Dam, pink) and log2(Dam::OGT/Dam, green) chromatin occupancy peaks (peak calling: find_peaks, q<0.01). (B) The distribution of the TPMs of genes occupied by OGT (green), Pcl (pink), and genes co-occupied by OGT and Pcl (purple). (C) Average log2(Dam::OGT/Dam; left) and $\log 2(D a m:: P c l / D a m)$ (right) signal on a CD (gray) and SD (green and pink, respectively) diet centered at OGT and Pcl co-occupied peaks. (D) Average CATaDa signal on a CD (gray) and SD (yellow) centered at OGT and Pcl co-occupied peaks with (right) and without (left) OSMI-1. (E) iPAGE pathway analysis of genes co-occupied by OGT and Pcl. Text in blue represents neural GO terms and purple represents regulatory GO terms. (F) L2fC of DEGs at SD7, $\mathrm{Pcl}^{\mathrm{c} 429}$, and OSMI-1, shades of red represents varying positive $12 \mathrm{fc}$ and shades of gray represents varying negative $12 \mathrm{fc}$.

Figure 6: Nutrigenomic responses in the lingual epithelium of rats fed high dietary sucrose.

(A) Schematic of rat sucrose feeding paradigm. Rats were fed either chow and water (gray) or chow and water supplemented with $30 \%$ sucrose in their water. (B) Volcano representation of genes differentially expressed (blue) in the tongue epithelia of rats on a chow+sucrose versus chow diet ( $q<0.1$, Wald test). (C) GO terms associated with the DEG in tongue epithelium (FDR<0.05). (D) Overlap of differentially expressed genes in the 
tongue epithelia of rats fed sucrose+chow diet (blue) with a previously published OGT ChIP dataset (green). (E) OGT ChIP-seq read density centered at the TSS of tongue epithelia DEGs (green) and non-DEGs (gray).

(F) MTF2 ChIP-seq read density centered at the TSS of DEGs (pink) versus non-DEGs (gray). (G) Network of DEGs of tongue epithelia of rats fed a sucrose+chow diet that are also enriched in epithelial taste/progenitor cells. Shade of green circles represents the interaction confidence score. $(\mathbf{H})$ Model for the role of nutriepigenetic signaling and nutrient information in taste plasticity; HBP, Hexosamine Biosynthesis Pathway, $\mathrm{TF}$, transcription factor.

\section{Supplemental Figure Legends}

\section{Figure S1 -Related to Figure 1, OGT role in taste plasticity}

(A) Proboscis extension response (PER) to stimulation of the labellum with 30,10 , and $5 \%$ sucrose ( $x$ axis) of age-matched male Gr5a;tubulin-GAL80 ${ }^{\text {ts }}$ (green) and transgenic controls (shades of gray) on a control diet, $\mathrm{n}=$ 17-24. (B) Quantification of the number of sweet taste GFP-labeled cells in the labella of Gr64f;CD8-GFP flies crossed to $w 1118^{c s}$ (as control, gray), Gr64f;CD8-GFP>OGT ${ }^{R N A i}$ (light green), and Gr64f;CD8-GFP>OGT (dark green) on a control (circle) or sugar (square) diet for 7 days. $n=5-16$ probosces, no significance, Kruskal-Wallis Dunn's multiple comparisons, comparison to control diet of each genotype. (C) Gr5a>OGT RNAi (green) and transgenic controls (shades of gray) on a sugar diet supplemented with OSMI or vehicle, Kruskal-Wallis test with Dunn's multiple comparisons, comparisons to transgenic controls, $n=51-63$. (D) Taste responses ( $y$ axis) to stimulation of the labellum with 30,10 , and $5 \%$ sucrose ( $x$ axis) of age-matched male $w 1118^{c s}$ flies on a control (circle) or sugar (square) diet with vehicle (10\% DMSO) or 10 and 30uM OSMI-1. $\mathrm{n}=$ 14-17.Kruskal-Wallis Dunn's multiple comparisons, comparison to control diet of each genotype. In all panels, flies were on a control (circle) or sugar (square) diet for 7 days. Data are shown as means \pm SEM. ${ }^{* * *} P<$ $0.0001,{ }^{* *} P<0.001,{ }^{* *} P<0.01$, and ${ }^{*} P<0.05$.

Figure S2- Related to Figure 3, Effects of OGT activity on PRC2.1 nutrient-dependent occupancy

(A) Principal component analysis of normalized log2(Dam::Pcl/Dam) flies on CD+OSMI-1( gray) or SD+OSMI-1 (pink). (B) Overlap of log2(Dam::Pcl/Dam) chromatin occupancy peaks of CD+OSMl-1 (gray) and SD+OSMI-1 (pink) (find_peaks, q<0.01). (C) iPAGE pathway analysis of genes shared between log2(Dam::Pcl/Dam) peaks with and without OSMI-1. (D) iPAGE pathway analysis of genes specifically occupied by Dam::Pcl with or without OSMI-1.

\section{Figure S3- Related to Figure 4, OGT resides on the chromatin of the Gr5a+ neurons}

(A) Principal component analysis of normalized log2(Dam::OGT/Dam) flies on CD ( gray) or SD (green). (B) CATaDa from control diet flies at the sweet gustatory receptor Gr5a and the bitter gustatory receptor Gr66a. (C) 
Proportion of observed Dam::OGT consensus peaks allocated to their respective chromatin domains normalized to the expected proportions across the whole genome.

Figure S4- Related to Figure 4, Pathway enrichment analysis of OGT chromatin targets in the Gr5a+ neurons

iPAGE identification of pathways depleted (blue) or enriched (red) compared to background gene list from the Dam::OGT peaks on a control, sugar, and difference of sugar/control diet. Scale represents over-representation (red) or under-representation (blue) of genes within a specific bin for the corresponding GO term. Black outlined boxes represent $\mathrm{q}<0.05$.

Figure S5 - Related to Figure 5, OGT activity directs chromatin and transcriptional responses to the dietary environment

(A) Log2(Dam::OGT/Dam) on a control (black) and sugar (green) diet within a $50 \mathrm{~kb}$ window at cad and Ptx1, and a $100 \mathrm{~kb}$ window at scro. Replicates are superimposed. Pink traces are SD/CD fold changes (top). (B) Schematic of Flag tagged ribosomes expressed in the Gr5a+ cells and isolated by immunoprecipitation. (C) Principal component analysis of Gr5a > UAS-Rp/3-3XFLAG flies on a control (gray) or sugar (green) diet for 7 days supplemented with OSMI-1. $n=3$ replicates per condition. (D) $\log _{2} f c(G r 5 a / I n p u t)$ of the genes Gr5a, Gr64f, Gr64a, Ir56D, Gr32a, and Gr66a. (E) Volcano plot representing differential expression in the Gr5a+ neurons of age-matched male Gr5a>UAS-Rp/3-3XFLAG flies on a sugar diet for 7 days supplemented with OSMI-1. $n=3$ replicates per condition. Nonsignificant genes are in black, and genes with $q<0.1$ (Wald test) are in green. (F) Log2 fold changes for candidate gene targets of Cad, Ptx1, and scro at SD7 (teal), Pclc429 (pink) and OSMI-1 fed flies at SD7 (green).

\section{Figure S6- Related to Figure 5, Pathway enrichment analysis of genes reverted or unchanged by}

\section{OSMI-1}

iPAGE identification of pathways depleted (blue) or enriched (red) compared to background gene list from genes with positive or negative log2 fold changes on SD+OSMI-1. Scale represents over-representation (red) or under-representation (blue) of genes within a specific bin for the corresponding GO term. Black outlined boxes represent $\mathrm{q}<0.05$.

Figure S7- Related to Figure 6, Nutrigenomic responses in the lingual epithelium of rats fed high dietary sucrose

(A) Principal component analysis of rats fed either chow and water (gray) or chow and water supplemented with $30 \%$ sucrose in their water (blue). $n=4$ replicates per condition. (B) The normalized levels of the reads 
bioRxiv preprint doi: https://doi.org/10.1101/2021.12.17.473205; this version posted December 19,2021 . The copyright holder for this preprint (which was not certified by peer review) is the author/funder, who has granted bioRxiv a license to display the preprint in perpetuity. It is made available under aCC-BY-NC-ND 4.0 International license.

associated with genes enriched in the taste epithelium (Krt14, Krt5, Tp63) vs mesenchyme (Vim, acta5). (C-D) H3K27me3 (C) and H3K27ace (D) ChIP-seq read density centered at the TSS of tongue epithelia DEGs (red and green respectively) and non-DEGs (gray). (E) GO terms associated with DEGs of tongue epithelia of rats fed a sucrose+chow diet that are also co-occupied by OGT and MTF2. 